\title{
Renormalization of Models with Radiative Mass Generation
}

\author{
G. Bandelloni, C. Becchi, A. Blasi, and R. Collina \\ Istituto di Scienze Fisiche dell'Universitá di Genova, Genova, Italy, and \\ Istituto Nazionale di Fisica Nucleare, Sezione di Genova, Genova, Italy
}

\begin{abstract}
The mechanism of radiative mass generation is discussed by means of a simple model with spontaneously broken symmetry. We show how this phenomenon induces an infrared breakdown of the usual perturbative approach and proceed to identify a set of renormalization prescriptions allowing the construction of a new perturbation theory in which the Ward identities of the model are maintained. The original pathologies are reflected in the appearance of square roots and logarithms of the expansion parameter $\hbar$.
\end{abstract}

\section{Introduction}

In the last few years attention has been paid to the mechanism of mass generation induced by radiative corrections [1]. The interest is largely due to the phenomenological insights this mechanism may provide such as, for instance, the nature of electron's [2] and pion's mass [1] or the possibility of breaking the C.P. Symmetry [3] at the quantum level.

The phenomenon typically arises when in a classical field model (tree approximation) there are constraints which imply the "accidental" vanishing of some particle masses. This is known to happen, due to the particular "representation content" of the scalar multiplet and to the condition of renormalizability by power counting, in some models with spontaneous symmetry breaking and in gauge models exhibiting the Higgs Kibble [4] (H.K.) mechanism. For example in some H.K. gauge models the classical potential energy of the scalar fields (where no couplings with other fields are present) may be invariant with respect to a larger symmetry group, i.e., one which contains the gauge group as a proper subgroup, this larger symmetry being violated by the other terms of the Lagrangian. It follows that there are "accidentally" more Goldstone bosons than those implied by the symmetry of the model which are not reabsorbed by the H.K. mechanism. These residual massless bosons are named pseudo Goldstone bosons (P.G.B.) [1].

Outside of the H.K. mechanism, some non-gauge models with spontaneous symmetry breaking, may also show radiative mass generation when part of the 
lagrangian is invariant for a symmetry greater than the oririnal one and this larger group is broken by couplings which do not provide, due to the particular choice of the vacuum state, any mass term to the Goldstone bosons. If so there may be more massless bosons than the number predicted by the Goldstone theorem [5].

Accidentally massless fermions may also appear in other models with symmetry breaking, when the "representation content" and the power counting requirement impose that some fermion fields be decoupled, at the classical level, from those boson fields which acquire a non-vanishing vacuum expectation value.

In all these cases the radiative corrections are expected to generate mass terms for the particles which remain accidentally massless; when this happens the perturbation theory breaks down since, as well shall see, the Feynman amplitudes develop I.R. divergences.

Now, even if the phenomenological interest in these models is focused on the mere existence of such a mechanism of radiative mass generation, we are still faced with the academic-theoretical problem of interpreting and possibly solving the perturbative breakdown, having in mind that a detailed description of this phenomenon may give some general hints about the implications of the perturbative infrared pathologies.

Our task in this paper will be the construction of a new scheme allowing a full perturbative treatment of the models with radiative mass generation and of their symmetry properties hence solving the mentioned perturbative breakdown.

We shall adopt as a reference a very simple model with spontaneous symmetry breaking (shown in Sect.2) by which we shall illustrate the construction of a covariant perturbation theory taking into account, since the beginning (i.e., at the propagator level), the radiatively generated masses. This perturbation theory will be analyzed in the framework of an I.R. extension of the B.P.H.Z. renormalization scheme, [6], [7] showing that its formal expansion parameter is the square root of $\hbar$ and that it maintains the loop ordering of the Gell Mann-Low series (Sect. 3).

Finally we shall prove that our scheme also allows the implementing to the quantum level of the Ward identities which characterize the symmetry properties of the model (Sect. 4).

The appendix contains the more technical aspects and developments of our approach.

\section{Analysis of a Simple Model}

The phenomenon of radiative mass generation may appear, as discussed in the introduction, in many different ways both in models with spontaneous symmetry breaking or in gauge models and may involve both boson or fermion fields. Out of all these possibilities we shall select, and employ as our standard reference, a simple Goldstone-type model where the spontaneous breaking of the symmetry associated with the $O(3) \times U(1)$ group generates P.G.B. particles.

The model is built with a five complex component field whose real and imaginary parts separately transform according to the five dimensional irreducible representation of the rotation group $O(3)$ (angular momentum equal to two). The $U(1)$ transformation multiplies the field with a phase factor. In other words the 
components of the field can be organized in a traceless symmetric $3 \times 3$ matrix whose elements are:

$$
\Phi_{i j}(x) / \varphi_{i j}(x)+i \psi_{i j}(x) \quad i, j=1,2,3
$$

and which behave under infinitesimal transformation as

$$
\begin{aligned}
& \delta \varphi_{i j}(x)=\left[\omega^{l} t^{l}, \varphi(x)\right]_{i j}+\omega^{0} \psi_{i j}(x), \\
& \delta \psi_{i j}(x)=\left[\omega^{l} t^{l}, \psi(x)\right]_{i j}-\omega^{0} \varphi_{i j}(x)
\end{aligned}
$$

where $\omega^{l}(l=1,2,3), \omega^{0}$ are respectively the parameters of the $O(3)$ and $U(1)$ groups and $t_{i k}^{l}=\varepsilon^{i l k}$ is the totally antisymmetric tensor.

The most general renormalizable lagrangian invariant under the transformations in Eqs. (2) contains terms quadratic and quartic in the fields components, $\varphi_{i j}(x)$ and $\psi_{i j}(x)$, the cubic contributions being excluded by the $U(1)$ invariance. In particular the potential energy of the model has only one quadratic invariant term which is

$$
\operatorname{Tr}\left(\Phi \Phi^{\dagger}\right) \equiv\left\langle\Phi \Phi^{\dagger}\right\rangle
$$

while the contribution of the quartic invariants is limited to three linearly independent terms since the four invariants

$$
\left\langle\Phi \Phi^{\dagger}\right\rangle^{2},\left\langle\Phi^{2}\right\rangle\left\langle\left(\Phi^{\dagger}\right)^{2}\right\rangle,\left\langle\Phi^{2}\left(\Phi^{\dagger}\right)^{2}\right\rangle,\left\langle\left(\Phi \Phi^{\dagger}\right)^{2}\right\rangle
$$

obey the relation [8]

$$
4\left\langle\Phi^{2}\left(\Phi^{\dagger}\right)^{2}\right\rangle+2\left\langle\left(\Phi \Phi^{\dagger}\right)^{2}\right\rangle-\left\langle\Phi^{2}\right\rangle\left\langle\left(\Phi^{\dagger}\right)^{2}\right\rangle-2\left\langle\Phi \Phi^{\dagger}\right\rangle^{2}=0 .
$$

Hence, in terms of the real components $\varphi_{i j}$ and $\psi_{i j}$ of the field, the potential energy $V$ can be written as

$$
\begin{aligned}
V= & -\frac{a}{2}\left\langle\varphi^{2}+\psi^{2}\right\rangle+\frac{\mu}{4 !}\left\langle\varphi^{2}+\psi^{2}\right\rangle^{2} \\
& -\frac{\lambda}{4 !}\left\langle\left(\varphi^{2}+\psi^{2}\right)^{2}\right\rangle-\frac{\eta}{4 !}\left\langle[\varphi, \psi]^{2}\right\rangle .
\end{aligned}
$$

We shall investigate the model only for positive values of the parameters $\mu, \lambda, \eta$; with this constraint and making use of the inequalities

$$
\begin{aligned}
& \frac{1}{3}\left\langle\varphi^{2}+\psi^{2}\right\rangle^{2} \leqq\left\langle\left(\varphi^{2}+\psi^{2}\right)^{2}\right\rangle \leqq \frac{1}{2}\left\langle\varphi^{2}+\psi^{2}\right\rangle^{2} \\
-\frac{1}{2}\left\langle\varphi^{2}+\psi^{2}\right\rangle^{2} & \left.\leqq\langle\varphi, \psi]^{2}\right\rangle \leqq 0
\end{aligned}
$$

the requirement that $V$ be bounded from below is easily seen to imply

$$
\mu>\frac{\lambda}{2} \text {. }
$$

Of course the spontaneous symmetry breaking mechanism takes place only for positive values of the parameter $a$ and the fundamental state of the system coincides with an absolute minimum of the potential energy. Setting

$$
f=\sqrt{\left\langle\Phi \Phi^{\dagger}\right\rangle}
$$


is clearly seen by the inequalities (7), that the potential energy reaches a minimum, for fixed $f$, when

$$
\begin{aligned}
\langle[\varphi, \psi]\rangle & =0, \\
\left\langle\left(\varphi^{2}+\psi^{2}\right)^{2}\right\rangle & =\frac{1}{2} f^{4}
\end{aligned}
$$

and the absolute minimum is obtained for

$$
f=\sqrt{\frac{3 ! a}{\mu-\frac{\lambda}{2}}} \equiv F
$$

The peculiar aspect of this model, and consequently its pathology, lies in the fact that the solutions of Eqs. (10) are not connected one to the other by the symmetry group transformations. This property is shown explicitly by the choices

$$
\begin{aligned}
& \Phi_{1}=\frac{F}{\sqrt{6}}\left(\begin{array}{rrr}
1 & 0 & 0 \\
0 & 1 & 0 \\
0 & 0 & -2
\end{array}\right) \\
& \Phi_{2}=\frac{F}{\sqrt{2}}\left(\begin{array}{llr}
1 & 0 & 0 \\
0 & 1 & 0 \\
0 & 0 & 0
\end{array}\right)
\end{aligned}
$$

which both solve Eqs. (10).

The set of field configurations of minimal energy is individuated by the orbit spanned by a linear superposition of $\Phi_{1}$ and $\Phi_{2}$

$$
\Phi_{0}(\theta)=\frac{F \cos \theta}{\sqrt{6}}\left(\begin{array}{rrr}
1 & 0 & 0 \\
0 & 1 & 0 \\
0 & 0 & -2
\end{array}\right)+\frac{F \sin \theta}{\sqrt{2}}\left(\begin{array}{lll}
1 & 0 & 0 \\
0 & 1 & 0 \\
0 & 0 & 0
\end{array}\right) \text { for }|\theta| \leqq \frac{\pi}{6}
$$

Therefore we can picture the situation as follows: while in the usual Goldstone type models the equilibrium configurations of the system are located along valleys which are covered by the symmetry group transformations, in our class of models such valleys acquire some extra degree of freedom (one in the case at hand) to which may be associated P.G.B. particles, i.e., massless particles which become massive through the radiative corrections. To illustrate further the dynamical mass generation we emphasize that the width of the valleys, which stays fixed along the orbit, indeed varies with $\theta$ in Eq. (13). Furthermore, forgetting for the time being the space dependence of the field and taking it to be defined at a single space point, we observe that the lowest energy eigenvalue associated with the transverse motions in the valleys decreases when the width of the valley increases. Hence, taking into account this quantum effect by means of a Born-Oppenheimer approximation, we may conclude that the system will feel an attractive force toward the widest region of the valley, and the elastic constants of this force will play the role, in the full quantum model, of the masses of the P.G.B. particles. 
Going back to our model we notice that the zero point energy of the transverse oscillations around the field configuration in Eq. (13) is given by

$$
\begin{aligned}
K(\theta)= & 2 F\left\{\sqrt{\frac{\lambda}{3} \cos ^{2} \theta+\eta \sin ^{2} \theta}+\sqrt{\frac{\lambda}{3} \cos ^{2}\left(\theta+\frac{\pi}{3}\right)+\eta \sin ^{2}\left(\theta+\frac{\pi}{3}\right)}\right. \\
& \left.+\sqrt{\frac{\lambda}{3} \cos ^{2}\left(\theta-\frac{\pi}{3}\right)+\eta \sin ^{2}\left(\theta-\frac{\pi}{3}\right)}\right\}
\end{aligned}
$$

from which we can conclude that the equilibrium point of the system is given by the configuration with $\theta=0$ if $\frac{\lambda}{3}<\eta$ and $\theta= \pm \frac{\pi}{6}$ if $\frac{\lambda}{3}>\eta$. For $\frac{\lambda}{3}=\eta, K(\theta)$ in Eq. (14) is constant and hence we have a $\theta$-indifferent equilibrium position. The explanation of this lies in the fact that for $\frac{\lambda}{3}=\eta$ the symmetry group of the model is no longer $O(3) \times U(1)$, but the potential energy has become invariant under the larger group $O(5) \times U(1)$, and no P.G.B. phenomenon arises.

Considering from now on only the values $\frac{\lambda}{3}<\eta$, and performing the field translation

$$
\begin{aligned}
& \varphi_{i j}(x) \rightarrow \varphi_{i j}(x)+\bar{\varphi}_{i j} \\
& \psi_{i j}(x) \rightarrow \psi_{i j}(x)
\end{aligned}
$$

where

$$
\bar{\varphi}=\Phi_{0}(0)=\frac{F}{\sqrt{6}}\left(\begin{array}{rrr}
1 & 0 & 0 \\
0 & 1 & 0 \\
0 & 0 & -2
\end{array}\right)
$$

we obtain the lagrangian of the system, in pseudoeuclidean metric

$$
\begin{aligned}
\mathscr{L}= & -\frac{1}{2}\left\langle\partial_{\mu} \varphi(x) \partial^{\mu} \varphi(x)+\partial_{\mu} \psi(x) \partial^{\mu} \psi(x)\right\rangle \\
& +\frac{a}{2}\left\langle(\varphi(x)+\bar{\varphi})^{2}+\psi^{2}(x)\right\rangle-\frac{\mu}{4 !}\left\langle(\varphi(x)+\bar{\varphi})^{2}+\psi^{2}(x)\right\rangle^{2} \\
& +\frac{\lambda}{4 !}\left\langle\left[(\varphi(x)+\bar{\varphi})^{2}+\psi^{2}(x)\right]^{2}\right\rangle+\frac{\eta}{4 !}\left\langle[\varphi(x)+\bar{\varphi}, \psi(x)]^{2}\right\rangle-C
\end{aligned}
$$

and

$$
\left.\mathscr{L}(\varphi)\right|_{\Phi=0}=\left.\frac{\partial \mathscr{L}}{\partial \varphi}\right|_{\Phi=0}=0 .
$$

Due to the choice in Eq. (16), the lagrangian in Eq. (17) is still invariant under the rotations around the $t^{3}$ axis; it is therefore natural to choose a new basis for the fields on which the generator of the $t^{3}$ rotation is diagonal. 
Defining the matrices

$$
\begin{array}{ll}
\lambda_{0}=\frac{1}{\sqrt{6}}\left(\begin{array}{rrr}
1 & 0 & 0 \\
0 & 1 & 0 \\
0 & 0 & -2
\end{array}\right) ; \quad \lambda_{1}^{-}=\frac{1}{2}\left(\begin{array}{rrr}
0 & 0 & 1 \\
0 & 0 & -i \\
1 & -i & 0
\end{array}\right) ; \quad \lambda_{1}^{+}=\frac{1}{2}\left(\begin{array}{rrrr}
0 & 0 & 1 \\
0 & 0 & i \\
1 & i & 0
\end{array}\right) ; \\
\lambda_{2}^{-}=\frac{1}{2}\left(\begin{array}{rrr}
1 & -i & 0 \\
-i & 1 & 0 \\
0 & 0 & 0
\end{array}\right) ; \quad \lambda_{2}^{+}=\frac{1}{2}\left(\begin{array}{rrr}
1 & i & 0 \\
i & 1 & 0 \\
0 & 0 & 0
\end{array}\right)
\end{array}
$$

the field components can be written

$$
\begin{aligned}
& \varphi(x)=\varphi_{0}(x) \lambda_{0}+\varphi_{1}^{+}(x) \lambda_{1}^{-}+\varphi_{1}^{-}(x) \lambda_{1}^{+}+\varphi_{2}^{+}(x) \lambda_{2}^{-}+\varphi_{2}^{-}(x) \lambda_{2}^{+}, \\
& \psi(x)=\psi_{0}(x) \lambda_{0}+\psi_{1}^{+}(x) \lambda_{1}^{-}+\psi_{1}^{-}(x) \lambda_{1}^{+}+\psi_{2}^{+}(x) \lambda_{2}^{-}+\psi_{2}^{-}(x) \lambda_{2}^{+} .
\end{aligned}
$$

We further notice that the lagrangian in Eq. (17) is invariant under the inversion

$$
\begin{aligned}
& \varphi(x) \rightarrow \varphi(x), \\
& \psi(x) \rightarrow-\psi(x)
\end{aligned}
$$

which excludes the presence of couplings with an odd number of $\psi$ fields.

The mass spectrum of the particles in the theory is now easily computed from Eq. (17) and Eq. (11); we summarize the result in the following formulae:

$$
\begin{aligned}
& m_{\varphi_{0}}^{2}=-\left.\frac{\partial^{2} \mathscr{L}}{\partial \varphi_{0} \partial \varphi_{0}}\right|_{\Phi=0}=\frac{F^{2}}{3}\left(\mu-\frac{\lambda}{2}\right), \\
& m_{\psi_{1}^{ \pm}}^{2}=-\left.\frac{\partial^{2} \mathscr{L}}{\partial \psi_{1}^{+} \partial \psi_{1}^{-}}\right|_{\Phi=0}=\frac{F^{2}}{4 !}\left(\frac{\lambda}{3}+3 \eta\right), \\
& m_{\psi_{2}^{ \pm}}^{2}=-\left.\frac{\partial^{2} \mathscr{L}}{\partial \psi_{2}^{+} \partial \psi_{2}^{-}}\right|_{\Phi=0}=\frac{F^{2}}{18} \lambda, \\
& m_{\varphi_{1}^{ \pm}}^{2}=m_{\varphi_{\frac{1}{2}}^{ \pm}}^{2}=m_{\psi_{0}}^{2}=0 .
\end{aligned}
$$

Since the vacuum expectation value of the scalar fields in Eq. (15a) is along $\lambda_{0}$, the field components $\varphi_{1}^{ \pm}$are the Goldstone bosons relative to the $O(3)$ group and $\psi_{0}$ is the one associated with $U(1)$, while the fields $\varphi_{2}^{+}, \varphi_{2}^{-}$, Eq. (21a), appear as possible pseudo Goldstone bosons.

Having thus described the classical model and shown the possibility of the appearance of radiative mass generation, we devote the rest of this section to show how this phenomenon manifests itself in standard perturbation theory. A convenient way of compactifying this analysis is provided by the functional language, which we now briefly summarize. We indicate by

$$
\Gamma(\Phi)=\sum_{n=2}^{\infty} \frac{1}{n !} \int d x_{1} \ldots d x_{n} \Phi_{i_{1}}\left(x_{1}\right) \ldots \Phi_{i_{n}}\left(x_{n}\right) G_{i_{1} \ldots i_{n}}\left(x_{1} \ldots x_{n}\right)
$$

the generating functional of the proper amplitudes $G_{i_{1} \ldots i_{n}}\left(x_{1} \ldots x_{n}\right)$ i.e., those associated with diagrams having $n$ amputated external legs and which cannot be separated into two disconnected pieces by cutting a single line. We recall also that in general $\Gamma(\Phi)$ is a formal power series in $\hbar$ [9] whose first term (i.e., the $\hbar=0$ 
contribution which defines the classical limit) coincides with the classical action $\Gamma^{\mathrm{cl}}(\Phi)$ of the theory. The symmetry properties of the model at the classical level can be translated into functional form as

$$
\begin{aligned}
& \mathscr{W}^{l} \Gamma^{\mathrm{cl}}(\Phi)=\int d^{4} x\left[\left\langle(\varphi(x)+\bar{\varphi}) t^{l} \frac{\delta}{\delta \varphi(x)}\right\rangle+\left\langle\psi(x) t^{l} \frac{\delta}{\delta \psi(x)}\right\rangle\right] \Gamma^{\mathrm{cl}}(\phi)=0 \\
& \mathscr{W}^{0} \Gamma^{\mathrm{cl}}(\Phi)=\int d^{4} x\left[-\left\langle(\varphi(x)+\bar{\varphi}) \frac{\delta}{\delta \psi(x)}\right\rangle+\left\langle\psi(x) \frac{\delta}{\delta \varphi(x)}\right\rangle\right] \Gamma^{\mathrm{cl}}(\Phi)=0 .
\end{aligned}
$$

The standard procedure is to verify if the model admits a vertex functional $\Gamma(\Phi)$ satifying the W.I. in Eq. (23) to all order of perturbation theory; however in models with spontaneously broken symmetry it is convenient to rewrite the W.I. in a non integrated form in order to avoid amplitudes with external legs at zero momentum.

To be able to translate the W.I. in local form we have to define an extension of the lagrangian in Eq. (17) which is invariant for (local) gauge transformations. To this purpose we introduce a multiplet of classical gauge vector fields $\mathscr{A}_{\mu}^{\alpha}$ $(\alpha=0,1,2,3)$ corresponding to the infinitesimal generators of the $O(3)(\alpha=1,2,3)$ and $U(1)(\alpha=0)$ groups. The local gauge transformations for the $\Phi$ fields are given in Eq. (2) with the $\left\{\omega^{l}, \omega^{0}\right\} \equiv\left\{\omega^{\alpha}\right\}$ parameters depending upon the space-time point $x$, while the fields $\mathscr{A}_{\mu}^{\alpha}$ transform accordingly to

$$
\delta \mathscr{A}_{\mu}^{\alpha}(x)=\partial_{\mu} \omega^{\alpha}(x)+f^{\alpha \beta \gamma} \mathscr{A}_{\mu}^{\gamma}(x) \omega^{\beta}(x)
$$

where $f^{\alpha \beta \gamma}=\varepsilon^{\alpha \beta \gamma}$ when restricted to $O(3)$ and $f^{\alpha \beta \gamma}=0$ if it involves at least a $U(1)$ index.

The gauge invariant extension of the lagrangian is then obtained substituting the space-time derivatives with the corresponding covariant derivatives

$$
\begin{aligned}
& \partial_{\mu} \varphi_{i j}(x) \rightarrow D_{\mu} \varphi_{i j}(x)=\partial_{\mu} \varphi_{i j}(x)+\left[\mathscr{A}_{\mu}^{l} t^{l}, \varphi\right]_{i j}(x)+\mathscr{A}_{\mu}^{0}(x) \psi_{i j}(x) \\
& \partial_{\mu} \psi_{i j}(x) \rightarrow D_{\mu} \psi_{i j}(x)=\partial_{\mu} \psi_{i j}(x)+\left[\mathscr{A}_{\mu}^{l} t^{l}, \psi\right]_{i j}(x)-\mathscr{A}_{\mu}^{0}(x) \varphi_{i j}(x)
\end{aligned}
$$

and by adding terms depending upon the covariant tensors built from the $\mathscr{A}_{\mu}^{\alpha}$ fields. The corresponding classical action $\Gamma^{\mathrm{cl}}\left(\mathscr{A}_{\mu}^{\alpha}, \Phi\right)$ satisfies the following non integrated W.I.

$$
\begin{aligned}
\mathscr{W}^{\alpha}(x) \Gamma^{\mathrm{cl}}\left(\mathscr{A}_{\mu}^{\alpha}, \Phi\right)= & \left\{\partial_{\mu} \frac{\delta}{\delta \mathscr{A}_{\mu}^{\alpha}(x)}+f^{\alpha \beta \gamma} \mathscr{A}_{\mu}^{\gamma}(x) \frac{\delta}{\delta \mathscr{A}_{\mu}^{\beta}(x)}\right. \\
& +\delta^{\alpha l}\left[\left\langle(\varphi(x)+\bar{\varphi}) t^{l} \frac{\delta}{\delta \varphi(x)}\right\rangle+\left\langle\psi(x) t^{l} \frac{\delta}{\delta \psi(x)}\right\rangle\right] \\
& \left.+\delta^{\alpha 0}\left[-\left\langle(\varphi(x)+\bar{\varphi}) \frac{\delta}{\delta \psi(x)}\right\rangle+\left\langle\psi(x) \frac{\delta}{\delta \varphi(x)}\right\rangle\right]\right\} \Gamma^{\mathrm{cl}}\left(\mathscr{A}_{\mu}^{\alpha}, \Phi\right) .
\end{aligned}
$$

It is straightforward to verify that the $\mathscr{W}^{\alpha}(x)$ operators obey the commutation relations.

$$
\left[\mathscr{W}^{\alpha}(x), \mathscr{W}^{\beta}(y)\right]=\delta(x-y) f^{\alpha \beta \gamma} \mathscr{W}^{\gamma}(x)
$$


The functional apparatus we have introduced (at the price of being rather tedious) allows a standard discussion of the possible quantum extension of the model within the scheme of Ref. [10]. In theories involving only massive particles the W.I. of the type shown in Eq. (26) turn out to be renormalizable with the only exception of models containing the Adler Bardeen anomaly [11], whose uniqueness can be algebraically proved by means of the commutation relations Eq. (27). However this result cannot be directly transferred to our model which is affected by a new kind of anomaly of I.R. origin ${ }^{1}$.

We will now show how this anomaly is brought about by the radiative mass generation, trying to be as non technical as possible. To this purpose consider the one loop vertex amplitude $G_{211}$ with external legs $\varphi_{2}^{+} \varphi_{1}^{-} \varphi_{1}^{-}$[in the basis given in Eqs. (19)] and the difference $G_{11}-G_{22}$ between the one loop propagator corrections of the fields $\varphi_{1}^{ \pm}$and $\varphi_{2}^{ \pm}$respectively.

It is easy to show that these amplitudes, vanishing at the classical level, are connected by the W.I. and are not affected by either I.R. or U.V. singularities. An explicit one loop computation at zero external momenta yields

$$
\frac{\sqrt{2}}{F \sqrt{3}}\left(G_{22}-G_{11}\right)=G_{211}=i \frac{\pi^{2}}{48} \sqrt{\frac{2}{3}} F\left(\frac{\lambda}{3}-\eta\right)^{2} \ln \left(\frac{\lambda+9 \eta}{4 \lambda}\right) \text {. }
$$

Now this non-vanishing result originates the I.R. anomaly of our model.

Indeed in order to extend the theory to two or more loops it is necessary to introduce suitable counterterms which compensate the value at zero momentum of the amplitudes $G_{11}, G_{22}$ and $G_{211}$ otherwise, the Feynman integrals with two or more loops become I.R. divergent; for example the propagators of the massless fields $\left(\varphi_{1}^{ \pm}\right.$or $\left.\varphi_{2}^{ \pm}\right)$develop poles of arbitrarily high order at zero momentum. However these counterterms break the W.I. since they are not invariant under the symmetry group of the model. Hence we can only conclude that our model is not straightforwardly renormalizable.

Nevertheless, from a heuristic point of view, such a phenomenon may be interpreted as a perturbative accident. By this we mean that the $\varphi_{2}$ field, which after all is not a Goldstone boson, will become massive in the "true" model (i.e., we

were able to sum the perturbation series).
It may be useful to recall that the vanishing of the anomaly at $\frac{\lambda}{3}=\eta$ was to be expected since at this value the $\varphi_{2}^{ \pm}$fields become true Goldstone bosons.

\section{The New Perturbative Development}

We have just seen that the mass generation due to radiative corrections manifests itself as an I.R. anomaly in the usual perturbation theory and that it can be physically interpreted only in view of a summation of the perturbative series generating a mass term in the propagators of the P.G.B. particles.

We approach now the main problem of this paper, namely whether the summation can be embedded in a new covariant perturbation expansion which maintains, order by order, the symmetry of the theory itself. The first important

1 An analogous phenomenon is discussed in [12] in the framework of the supersymmetric O’Raifertaigh model 
difference between such a perturbative approach and the usual one should arise in the construction of the Fock space as a consequence of the finite mass renormalization of the P.G.B. particles, which in a formal non-covariant language, could be carried out by a singular Bogoliubov transformation [13].

From a covariant point of view, this is equivalent to adding to the free lagrangian the mass terms generated by the radiative corrections at least order and then define the Green functions by the well-known Gell-Mann Low formula endowed with suitable subtraction rules. Of course we still have to investigate whether the new theory fits into a perturbative scheme and if, within this scheme, the W.I. which characterize the symmetry of the system are maintained.

The rest of this section is devoted to the analysis of the new perturbative series while the questions concerning the W.I. will be treated in the next one.

In order to discuss the first point we recall that in the usual perturbative approach the $\hbar^{N}$ corrections to a given Green function correspond to a finite number of diagrams with at most $N$ loops. This is not guaranteed in the new theory where the square of the masses of the P.G.B. particles are proportional to $\hbar$. Indeed the behaviour of any amplitude for vanishing $\hbar$ will in general be affected by the mass singularities introduced in the Feynman integrals by the P.G.B. propagators.

A complete analysis of the nature of these mass singularities is quite involved and depends strongly on the momenta carried by the external vertices of the diagram; we will limit ourselves, as it is usual in models with massless particles, to external momenta which are Euclidean and non-exceptional in the sense of Symanzik [14], and where a given amplitude behaves, when the propagator mass $\mu$ vanishes, like [15]

$$
\sum_{n \geqq-\bar{n}} \mu^{n} C_{n m}\left(p_{1}, \ldots, p_{l}\right)(\ln \mu)^{m} .
$$

Hence in our theory the Feynman integral corresponding to a generic $N$ loop graph will depend on $\hbar$ as:

$$
\sum_{\substack{m \\ n \geqq-\bar{n}}} \hbar^{\frac{n}{2}+N} C_{n m}\left(p_{1}, \ldots, p_{l}\right)\left(\frac{\ln \hbar}{2}\right)^{m} .
$$

It is apparent from Eq. (30) that if the P.G.B. mass singularities completely hide the loop factor $\hbar^{N}$, we have to give up the possibility that the Green functions, built according to the new Feynman rules, have a formal perturbative expansion whose terms, ordered with the increasing powers of $\hbar$, correspond to a finite number of diagrams. We shall show in the following that this catastrophic event can only be avoided by a suitable set of renormalization conditions.

Consider, for example, the amplitude $G$ corresponding the one loop diagram in Fig. 1, where a cross stands for a $\hbar$ independent mass insertion and the dotted lines indicate the P.G.B. propagators.

A simple computation yields for the leading power

$G \propto \hbar^{-n+2}$ 
where $n$ counts the crosses. Hence in this example, the singularity in $\hbar$ increases with the number of the mass insertions.

We notice that, even excluding these mass counterterms, the diagram $G^{\prime}$ in Fig. 2

Fig. 1

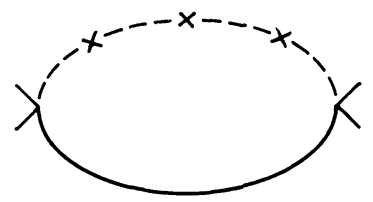

Fig. 2

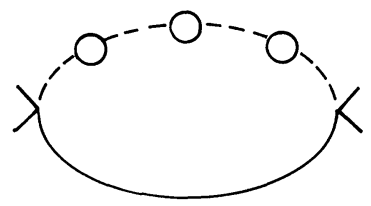

behaves like

$$
G^{\prime} \propto \hbar^{2}
$$

independently of the number of loops and provided there are at least two bubble insertions.

These simple examples already point out that all mass counterterms of the P.G.B. particles which do not vanish with a power greater than $\hbar$, must be excluded and likewise all P.G.B. propagator corrections at least order and zero momentum must be compensated with suitable mass counterterms. We shall show in the following that these prescriptions are sufficient to insure the existence of a suitable perturbation theory.

The translation of these qualitative considerations into precise renormalization conditions requires a few technical specifications, which we will now illustrate.

We shall renormalize our theory according to the B.P.H.Z. method in the extension given by Lowenstein and Zimmerman $[6,7]$ to include massless particles. The idea is to subtract the amplitudes at zero external momenta while keeping at a non zero value the masses in all the propagators of the subtracted diagram. Technically the procedure is to introduce, in all the propagators, a mass term depending upon a real parameter " $s$ ", $0 \leqq s \leqq 1$, and such that for $s=1$ the mass acquires its physical value, while it stays always positive when $0 \leqq s<1$.

In this framework the unsubtracted integral $I_{G}$ of any Feynman diagram $G$ assumes the form

$$
I_{G}(p, k, s)=\prod_{V \in G} P_{V}\left(\left\{l_{V}\right\}, s\right) \prod_{L \in G} \Delta_{L}\left(l_{L}, s\right)
$$

where the products run over the vertices $V$ and the lines $L$ of $G$ respectively. We have denoted by $\left\{l_{V}\right\}$ the set of momenta entering the vertex $V$ and by $l_{L}$ the momentum of the line $L$; of course both $l_{V}$ and $l_{L}$ are functions of the external $\{p\}$ and internal $\{k\}$ momenta of $G$. Furthermore $P_{V}$ is a polynomial in $l_{V}$ and the parameter $s$, and $\Delta_{L}\left(l_{L}, s\right)$, which is the product of the propagators associated of the line $L$, is of the form

$$
\Delta_{L}\left(l_{L}, s\right)=P_{L}\left(l_{L}, s\right) \prod_{i} \frac{1}{l_{L_{i}}^{2}+(s-1)^{2} M_{L_{i}}^{2}+m_{L_{i}}^{2}}
$$


where $m_{L_{i}}^{2} \geqq 0$ may be proportional to $\hbar$ and $M_{L_{i}}^{2}$ is independent from $\hbar$ and strictly positive for the massless and P.G.B. particles.

The corresponding renormalized integrand $R_{G}$ is defined by the well known forest formula

$$
R_{G}(p, k, s)=S_{G} \sum_{U \in \mathscr{F}_{G}}\left(-\tau_{\gamma} S_{\gamma}\right) I_{G}(U)
$$

where $\mathscr{F}_{G}$ is the set of forests of $G$ (families of non-trivial, non overlapping one particle irreducible (1 P.I.) subgraphs of $G), I_{G}(U)$ is the unsubtracted integrand parametrized with the variables of the smallest element $\lambda$ of $U$ and $S_{\gamma}$ is a substitution operator which shifts from the variables of $\lambda \in U$ to those of $\gamma \in U, \gamma \supset \lambda$. The details of this definition can be found in ref. [6].

Finally $\tau_{\gamma}$ is the subtraction operator which, for all the subdiagrams $\gamma$ different from the propagator corrections of the P.G.B. $\gamma_{2}$, is defined by

$$
1-\tau_{\gamma}=\left(1-t_{p^{\gamma}\left(s^{\gamma}-1\right)}^{\varrho(\gamma)-1}\right)\left(1-t_{p^{\gamma} s^{\gamma}}^{\delta(\gamma)}\right)
$$

while for these corrections we set

$$
1-\tau_{\gamma_{2}}=\left(1-t_{p(s-1)}^{0} t_{\hbar}^{1}\right)\left(1-t_{p s}^{2}\right)
$$

and

$$
t_{x_{1} \ldots x_{n}}^{d}=\left\{\begin{array}{l}
\text { Taylor polynomial about } x_{i}=0 \text { of degree } d \\
\text { when } d \geqq 0 \\
0 \text { otherwise }
\end{array}\right.
$$

The indices $\varrho(\gamma)$ and $\delta(\gamma)$ in the Eq. (36a) are given by:

$$
\begin{aligned}
& \varrho(\gamma)=4-\sum_{k} \underline{d}_{k}+\sum_{V_{\gamma}}\left(\varrho_{V_{\gamma}}-4\right), \\
& \delta(\gamma)=4-\sum_{k} \bar{d}_{k}+\sum_{V_{\gamma}}\left(\delta_{V_{\gamma}}-4\right)
\end{aligned}
$$

where the sum over $k$ refers to the external lines, $V_{\gamma}$ are the vertices of $\gamma$ and the following definitions have been used:

i) to each field of the theory are assigned U.V. dimensions $\bar{d}_{i}$ and I.R. dimensions $\underline{d}_{i}$ satisfying

$$
\begin{array}{r}
\overline{\operatorname{deg}}_{p s} \Delta_{L_{i j}}+4 \equiv \bar{D}_{L_{i j}}+4 \leqq \bar{d}_{i}+\bar{d}_{j} \\
\underline{\operatorname{deg}}_{p(s-1)} \Delta_{L_{i j}}+4 \equiv \underline{D}_{L_{i j}}+4 \geqq \underline{d}_{i}+\underline{d}_{j}
\end{array}
$$

where, for a generic function $F\left(x_{1} \ldots x_{n}, y_{1} \ldots y_{m}\right)$

$$
\begin{aligned}
& \overline{\operatorname{deg}}_{x_{1} \ldots x_{n}} F\left(x_{1}, \ldots, x_{n}, y_{1} \ldots y_{m}\right)=\bar{v} \\
& \text { if } \lim _{\lambda \rightarrow \infty} \lambda^{-\bar{v}} F\left(\lambda x_{1} \ldots \lambda x_{n}, y_{1} \ldots y_{m}\right) \neq 0, \infty \\
& \underline{\operatorname{deg}}_{x_{1} \ldots x_{n}} F\left(x_{1} \ldots x_{n}, y_{1} \ldots y_{m}\right)=\underline{y} \\
& \text { if } \lim _{\lambda \rightarrow 0} \lambda^{-\underline{v}} F\left(\lambda x_{1} \ldots \lambda x_{n}, y_{1} \ldots y_{m}\right) \neq 0, \infty
\end{aligned}
$$


for almost all values of $x_{1} \ldots x_{n}$ at fixed $y_{1} \ldots y_{m}$. Now, according to Eqs. (38), we can choose $\bar{d}_{i}=1$ and $\underline{d}_{i}=2$ for a massive boson, while for a massless one the I.R. dimension is $\underline{d}_{i}=1$ and the U.V. stays unchanged; we remark that the P.G.B. fields have $\underline{d}_{i}=2$ due to our choice of their propagators.

ii) To each vertex are associated the indices $\varrho_{V}$ and $\delta_{V}$ satisfying

$$
\begin{aligned}
\delta_{V} & \geqq \sum_{k} v_{k}(V) \bar{d}_{k}+\overline{\operatorname{deg}}_{p s} P_{V}(p, k, s) \\
& \equiv \sum_{k} v_{k}(V) \bar{d}_{k}+\bar{D}_{V} \\
\varrho_{V} & \leqq \sum_{k} v_{k}(V) \underline{d}_{k}+\underline{\operatorname{deg}}_{p(s-1)} P_{V}(p, k, s) \\
& \equiv \sum_{k} v_{k}(V) \underline{d}_{k}+\underline{D}_{V}
\end{aligned}
$$

where $v_{k}(V)$ counts the number of lines of the $k$-th type entering the vertex $V$ and $\varrho_{V} \leqq \delta_{V}$ except at must one vertex in the diagram for which $\varrho_{V}=\delta_{V}+1$ is allowed.

In particular the internal vertices of the diagrams, corresponding to the interaction lagrangian, will be assigned $\varrho_{V}=\delta_{V}=4$. This condition will also hold for the couplings of the external gauge fields $\mathscr{A}_{\mu}^{\alpha}$, which have been introduced in Sect. 2 in order to define the W.I. Eq. (26). These fields which appear only as external lines in the diagrams, are given the I.R. and U.V. dimension $\underline{d}_{\alpha}=\bar{d}_{\alpha}=1^{2}$. The subtraction indices $\varrho_{V}$ and $\delta_{V}$ of other possible external vertices corresponding to composite local operators (here after denoted with C.E.V.) will be specified when needed.

In the framework of these subtraction rules the interaction lagrangian $\mathscr{L}^{\text {int }}$ will be chosen as a formal power series [16] in the parameter $\hbar^{1 / 2}$

$$
\mathscr{L}^{\text {int }}=\mathscr{L}_{0}^{\text {int }}+\sum_{n=1}^{\infty} \hbar^{\frac{n+1}{2}} \mathscr{L}_{(n)}(\ln \hbar)=\mathscr{L}_{0}^{\text {int }}+\sum_{n=2}^{\infty} \mathscr{L}_{n}^{\text {int }}
$$

where $\mathscr{L}_{0}^{\text {int }}$ is deduced from the classical lagrangian of the theory by dropping the bilinear (free) part, and $\mathscr{L}^{\text {int }}$ is constrained from the following supplementary conditions :

A) Assigning to the fields a new I.R. dimension $\underline{d}_{i}^{0}$ by:

$$
\begin{array}{ll}
\underline{d}_{i}^{0}=1 & \text { for the P.G.B. } \\
\underline{d}_{i}^{0}=\underline{d}_{i} & \text { otherwise }
\end{array}
$$

which implies

$$
\underline{\operatorname{deg}}_{\hbar^{1 / 2} p(s-1)} \Delta_{L_{i j}} \equiv \underline{D}_{L_{i j}} \underline{d}_{i}^{0}+\underline{d}_{j}^{0}-4
$$

and setting

$$
\not D_{V} \equiv \underline{\operatorname{deg}}_{\hbar^{1 / 2} p(s-1)} P_{V}
$$

2 Note that, e.g., the derivative bilinear coupling of a gauge field and a spinless boson field needs no subtraction at all 
the vertices of $\mathscr{L}^{\text {int }}$ obey also the inequality

$$
\not D_{V}+\sum_{k} v_{k}(V) \underline{d}_{k}^{0}-4 \geqq 0 ;
$$

B) Denoting by $P_{V_{2}}$ the coupling of 2 P.G.B. fields, the condition

$$
\underline{\operatorname{deg}}_{\hbar^{1 / 2} p(s-1)} P_{V_{2}} \equiv \not_{P_{V_{2}}}>2
$$

must be satisfied.

A few comments are now appropriate: the subtraction rules introduced in Eq. (36a) are identical to those given in [6], while the one given in Eq. (36b) ensures that the P.G.B. propagator corrections of order $\hbar$ vanish at $p=0$ and $s=1$. Notice that the integrand of a P.G.B. propagator correction $\gamma_{2}$ depends on $\hbar$ according to

$$
I_{\gamma_{2}}=\hbar\left[I_{\gamma_{2}}^{(0)}+\sum_{n=2}^{\infty} \hbar^{\frac{n}{2}} I_{\gamma_{2}}^{(n)}\right]
$$

where $I_{\gamma_{2}}^{(0)}$ is built only with the vertices of $\mathscr{L}_{0}^{\text {int }}$.

Moreover the extrasubtraction for $\gamma_{2}$ does not affect the absolute convergence of the Feynman integrals since the integral of $I_{\gamma_{2}}^{(0)}$ is free from I.R. singularities. It is also important to realize, for the future, that the extra subtraction for $\gamma_{2}$ is completely equivalent to the introduction of a counterterm in the effective lagrangian, and hence it does not invalidate the quantum action principle as extended by Clark and Lowenstein to theories with massless particles [17].

Once specified the renormalization prescription for our model, we are able to evaluate the leading power in $\hbar$ for any subtracted Feynman amplitude. The detailed analysis is given in the Appendix; we here summarize the results in the following:

Theorem 1. Let $J_{A}$ be the amplitude corresponding to a proper Feynman diagram with $N$ loops and non exceptional, Euclidean external momenta; if $\Lambda$ contains no C.E.V. we have

$$
\underline{\operatorname{deg}}_{\hbar^{1 / 2}} J_{\Lambda} \geqq N+1
$$

otherwise

$$
\underline{\operatorname{deg}}_{\hbar^{1 / 2}} J_{\Lambda} \geqq N+2-\lambda_{\Lambda}
$$

where $\lambda_{A}$ is defined by

$$
\lambda_{\Lambda}=\sum_{\{\text {C.E.V.\} }} \lambda_{V} \equiv \sum_{\{\text {C.E.V.\} }}\left[\frac{\varrho_{V}}{2}\right]
$$

and the symbol $[x]$ stands for the least integer nearest to $x$.

Let us notice that possible $\hbar$ powers in the vertex constant of the C.E.V. have not been considered in the evaluation of Eq. (49) and hence their contribution, should be added to the r.h.s. of Eq. (49). Concerning the internal vertices, their $\hbar$ degree has been taken into account in Eq. (45); however, should it be greater than the one necessary to verify Eq. (45), the excess contribution is also added to the r.h.s. of Eqs. (48), (49). 
From these considerations we deduce that the substitution, in a diagram $\Lambda$, of a vertex constant with one of its higher order corrections in $\hbar$ or else the introduction of mass counterterms satisfying Eq. (46), increases the $\hbar$ degree of the graph itself. Thus the $\hbar^{1 / 2}$ degree of an amplitude $J_{\Lambda}$ with $N$ loops containing a vertex correction of degree $\chi>0$ is greater than or equal to $\chi-1+N$ or $\chi+N$ depending on whether the vertex is a P.G.B. mass correction or not. It is however shown in the Appendix that when $N=1$ the $\hbar^{1 / 2}$ degree of $\Lambda_{1}$ will be at least $\chi+1$ in both cases. Hence we have the following

Corollary. A generic proper Green function $G$ is a formal power series [16] in $\hbar^{1 / 2}$ of the type $e^{3}$

$$
G=G_{0}+\sum_{n=1}^{\infty} \hbar^{\frac{n+1}{2}} G_{(n)}(\ln \hbar)
$$

where $G_{(n)}$ has contributions from a finite number of diagrams with at most $n$ loops. Moreover the term $G_{(n)}(\ln \hbar)$ does not depend upon $\mathscr{L}_{\left(n^{\prime}\right)}(\ln \hbar)$ in Eq. (42) for $n^{\prime}>n$ and the only contributions from $\mathscr{L}_{(n)}(\ln \hbar)$ are tree approximation terms.

In the following Section we shall reword the result of this corollary and in particular Eq. (51) saying that the functional $\Gamma$, defined in Sect. 2 Eq. (22), is a formal power series in $\hbar^{1 / 2}$.

We conclude this section with a brief summary of the results; we have shown a way of interpreting the dynamical mass generation in a new perturbative framework, which maintains the usual feature of linking the perturbation series to the loop expansion.

The I.R. singularities due to the radiative mass generation manifest themselves through the appearance of non integer power of $\hbar$ and of $\ln \hbar$ terms in the coefficients of Eq. $(51)^{4}$. The peculiar dependence on $\hbar$ of diagrams with composite external vertices is due to the fact that such vertices may enhance the I.R. singularities of the corresponding graph.

\section{Discussion of the Ward Identities}

In this Section we discuss the possibility of building, in agreement with our perturbation theory, a quantum vertex functional $\Gamma$ satisfying the W.I. in Eq. (26). The renormalizability of our model will be analyzed by means of quite general techniques whose applicability to broken symmetry or gauge models is widely discussed in the literature [10]. This will also give hints about a possible renormalization scheme for the whole class of theories mentioned in the Introduction.

We shall base our analysis on an extension to theories with massless particles [17] of the Lam-Lowenstein Q.A.P. [19] which we now recall in a simplified version suitable for linear symmetry transformations of the fields [20].

3 For what concerns the external momenta dependence of $G_{(n)}$ we note that, since these are Euclidean non exceptional, the expression in Eq. (51) is meant to hold almost everywhere for the Fourier transform of $G$

4 This phenomenon is somewhat analogous to the one discussed by K. Symanzik in [18] 
Consider the local field transformations

$$
\begin{aligned}
& \Phi_{i}(x) \rightarrow \Phi_{i}(x)+\lambda(x)\left[r_{i j}\left(\Phi_{j}(x)+F_{j}\right)\right] \\
& \quad=\Phi_{i}(x)+\lambda(x) \delta_{x} \Phi_{i}(x)
\end{aligned}
$$

where $\Phi_{i}(x)$ stands collectively for the quantized fields $\varphi_{i}, \psi_{i}$ and the external gauge fields $\mathscr{A}_{\mu}^{\alpha}$; the corresponding variation of the connected Green function $\left\langle\prod_{i=1}^{n} \Phi_{i}\left(x_{i}\right)\right\rangle_{+}$can be expressed in terms of the insertion of suitable normal product operators as

$$
\begin{aligned}
& \left\langle\sum_{j=1}^{n} \prod_{i=1}^{j-1} \Phi_{i}\left(x_{i}\right) \delta\left(x-x_{j}\right) \delta_{x_{j}} \Phi_{j}\left(x_{j}\right) \prod_{i=j+1}^{n} \Phi_{i}\left(x_{i}\right)\right\rangle_{+} \\
& =\left\langle\sum_{k} c_{k} N_{\varrho_{k}}^{\delta_{k}}\left[Q_{k}(x)\right] \prod_{i=1}^{n} \Phi_{i}\left(x_{i}\right)\right\rangle_{+} .
\end{aligned}
$$

Here $Q_{k}(x)$ is a generic polynomial in the fields $\Phi_{i}$, their derivatives and the $s$ parameter and the symbol $N_{\varrho_{k}}^{\delta_{k}}$ indicates the insertion into the Green function of the vertex corresponding to $Q_{k}(x)$ equipped with I.R. and U.V. subtraction indices $\varrho_{k}$ and $\delta_{k}$. These indices are related to the dimensionality of the naive variations of the vertices of the lagrangian and are constrained by Eqs. (40) and the condition $\varrho_{k} \leqq \delta_{k}+1$. In our case the index $\varrho_{k}$ may assume the values $3,4,5$ and $\delta_{k}$ the values 3 and 4.

By means of the Zimmermann identities $[21,16]$, establishing linear relations among the N.P.O. with different indices, the r.h.s. of Eq. (53) can be expressed in terms of a unique insertion with infrared index $\varrho$ equal to the smallest of the $\varrho_{k}$ 's and U.V. one $\delta$ equal to the largest of the $\delta_{k}$ 's. Furthermore any $s-1$ dependent term in this insertion can be isolated into a special vertex which does not contribute to the Green functions at $s=1^{5}$.

These considerations applied to the vertex functional of our model yield:

$$
\mathscr{W}^{\alpha}(x) \Gamma=\left.N_{3}^{4}\left[\Delta^{\alpha}(x)\right] \Gamma\right|_{s=1}+O(s-1)
$$

where the symbol $O(s-1)$ stands for the insertion of the N.P.O. vanishing at $s=1$.

At this point we open a short parenthesys to clarify a few questions. First of all the coefficients appearing in the operator $\mathscr{W}^{\alpha}(x)$ will be in general formal power series in $\hbar^{1 / 2}$; in spite of this, to avoid unessential complications we shall consider $\mathscr{W}^{\alpha}(x)$ as independent from $\hbar$. Secondly the functional $\mathscr{W}^{\alpha}(x) \Gamma$ (i.e., its Fourier transform) is a formal power series in $\hbar^{1 / 2}$ in the sense discussed at the end of Sect. 3 , and, due to the simplifying assumption for $\mathscr{W}^{\alpha}(x)$, its $n$-th order term is directly obtained by the application of the operator $\mathscr{W}^{\alpha}(x)$ to the corresponding term of $\Gamma$. Notice that this is the main reason to discuss the local W.I. instead of the integrated ones.

Going back to Eq. (54), we see that substituting on the 1.h.s. the tree approximation expression of $\Gamma$ yields on the r.h.s. contributions which either are proportional to $\hbar$, as those coming from the masses introduced in the P.G.B.

5 These N.P.O. are described by Clark and Lowenstein [16] introducing the symbol $N_{e}^{\delta}[]_{\omega}$ where the presence of the positive integer $\omega$ indicate that the contribution vanishes at least as $(s-1)^{\omega}$ 
propagators, or vanish at $s=1$ as those originated from the mass terms introduced to disentangle I.R. from U.V. singularities. Now, while the $\hbar$ proportional terms will hopefully compensate analogous contributions from the one loop diagrams (which requires a suitable choice of the P.G.B. masses), the $s-1$ dependent terms survive even in the limit $\hbar \rightarrow 0$ and are not compensable by the radiative corrections. It follows that the W.I. in Eq. (26) may only hold at the point $s=1$ where the original symmetry is restored. For these reasons our analysis will concern only the compensability of the first term on the r.h.s. of Eq. (54). In other words the renormalizability of the W.I. will be insured by proving the following theorem:

Theorem 2. The equation $N_{3}^{4}\left[\Delta^{\alpha}(x)\right] \Gamma=0$ can be solved in terms of the formal power series for $\mathscr{L}^{\text {int }}$ given in Eq. (41).

To arrive at this result we proceed by induction, i.e.:

a) we first show that

$$
N_{3}^{4}\left[\Delta^{\alpha}(x)\right] \Gamma=O(\hbar)^{6}
$$

is solved at $s=1$ by a choice of $\mathscr{L}_{0}$ corresponding to a classical invariant lagrangian.

b) By the inductive hypothesis that

$$
N_{3}^{4}\left[\Delta^{\alpha}(x)\right] \Gamma=O\left(\hbar^{\frac{N}{2}}\right)
$$

is solvable in terms of the coefficients of $\mathscr{L}^{\text {int }}$ up to the power $\frac{N-1}{2}$ we shall prove that

$$
N_{3}^{4}\left[\Delta^{\alpha}(x)\right] \Gamma=O\left(\hbar^{\frac{N+1}{2}}\right)
$$

has solutions in terms of the coefficients of $\mathscr{L}^{\text {int }}$ up to the power $\frac{N}{2}$.

To check the step (a) of our procedure we isolate in the 1.h.s. of Eq. (54) the tree approximation contributions from the ones with at least one loop and observe that the former give a term $O(\hbar)$ provided the theory is built at $s=1$ from a symmetric $\mathscr{L}_{0}$, while the latter are themselves $O(\hbar)$.

Concerning step (b), we first analyze in more detail the structure of the r.h.s. of Eq. (54) to exploit all the consequences of the validity of the induction hypothesis Eq. (56) and then proceed to a reformulation more suitable to our purposes. From Eq. (54) it follows that $N_{3}^{4}\left[\Delta_{\alpha}(x)\right] \Gamma$ is a formal power series in $\sqrt{\hbar}$ which by the inductive hypothesis in Eq. (56) has the form

$$
N_{3}^{4}\left[\Delta^{\alpha}(x)\right] \Gamma=\sum_{n=N}^{\infty} \hbar^{\frac{n}{2}} d_{n}^{\alpha}(\ln \hbar, x) .
$$

Taking into account the result of Theorem 1, we have that the lowest order terms on the r.h.s. of Eq. (58), i.e.,

$$
\hbar^{\frac{N}{2}} d_{N}^{\alpha}(\ln \hbar, x) \equiv \Delta_{N}^{\alpha}(x)
$$

6 We say, as usual, that a formal power series is $O\left(\hbar^{v}\right)$ if all the coefficients of the powers smaller than $v$ vanish identically 
come from tree graphs and hence $\Delta_{N}^{\alpha}(x)$ is a polynomial in the fields and their derivatives. Indeed a diagram with $M(M>0)$ loops and the insertion of a $N_{3}^{4}$ vertex (for which $\lambda_{V}=1$ ) has a $\hbar^{1 / 2}$ degree which is always greater than that of the vertex itself. We can thus say that Eq. (57) is equivalent to

$$
\Delta_{N}^{\alpha}(x)=0 \text {. }
$$

The solvability of this equation, in terms of the coefficients of order $\hbar^{N / 2}$ of $\mathscr{L}^{\text {int }}$, is a direct consequence of the following Lemma, proved at the end of this Section:

Lemma. In the hypothesis given in Eq. (56), an arbitrary choice of the $\hbar^{N / 2}$ order coefficients of $\mathscr{L}^{\text {Int }}$ gives

$$
\Delta_{N}^{\alpha}(x)=\mathscr{W}^{\alpha}(x) \hat{\Delta}_{N}
$$

where

$$
\hat{\Delta}_{N}=\int d x \hat{\Delta}_{N}(x)
$$

and $\hat{\Delta}_{N}(x)$ is a vertex with U.V. dimensions $\leqq 4$ and I.R. dimensions $\geqq 4$.

To show how Eq. (60) follows from the Lemma, let us remark that from Eqs. (54), (56), (59) we have

$$
\mathscr{W}^{\alpha}(x) \Gamma=\Delta_{N}^{\alpha}(x)+O\left(\hbar^{\frac{N+1}{2}}, s-1\right)
$$

and, isolating on the 1.h.s. of this expression the terms of order $\hbar^{\frac{N}{2}}$ (which we will indicate by a subscript $N$ ), we can write

$$
\left(\mathscr{W}^{\alpha}(x) \Gamma\right)_{N}=\mathscr{W}^{\alpha}(x) \Gamma_{N} \equiv \mathscr{W}^{\alpha}(x) \int d y \mathscr{L}_{N}^{\text {lnt }}(y)+Q_{N}^{\alpha}\left(\mathscr{L}_{v}^{\text {lnt }}, x\right), \quad v<N .
$$

On the r.h.s. of Eq. (64) we have separated the tree approximation contributions from the radiative corrections $Q_{N}^{\alpha}$ which, according to the corollary of Theorem 1 , depend from $\mathscr{L}_{v}^{\text {nt }}$ for $v<N$. Comparing Eq. (63) with Eq. (64) we obtain

$$
\Delta_{N}^{\alpha}(x)=\mathscr{W}^{\alpha}(x) \int d y \mathscr{L}_{N}^{\text {lnt }}(y)+Q_{N}^{\alpha}\left(\mathscr{L}_{v}^{\text {lnt }}, x\right),
$$

so that the further substitution of Eq. (61) in Eq. (65) yields

$$
Q_{N}^{\alpha}\left(\mathscr{L}_{v}^{\text {lnt }}, x\right)=\mathscr{W}^{\alpha}(x) \int \hat{Q}_{N}\left(\mathscr{L}_{v}^{\text {lnt }}, y\right) d y
$$

and hence Eq. (60) is solved by the choice

$$
\mathscr{L}_{N}^{\text {Int }}(x)=-\hat{Q}_{N}\left(\mathscr{L}_{v}^{\text {lnt }}, x\right)
$$

which, taking into account the I.R. and U.V. dimensions of $\hat{\Delta}_{N}$, is easily shown to be compatible with the constraints appearing in Eq. (45).

Let us also remark that the possible P.G.B. mass correction terms contained in $\hat{Q}_{2}$ cannot be introduced into $\mathscr{L}_{2}^{\text {Int }}$ but they must be compensated by a suitable redefinition of the P.G.B. propagators. This procedure is legitimate since, as shown in Sect. 2, the diagrams which contribute to such mass corrections in $\hat{Q}_{2}$ are free from I.R. singularities which implies that $\hat{Q}_{2}$ does not depend on the values of the P.G.B. masses. 
The proof of the lemma can now be completed employing the techniques of [10]. The first step, in this approach, is the introduction of a functional description of diagrams with $\Delta_{N}^{\alpha}(x)$ vertices. This is carried out, as usual, by adding external $\beta^{\alpha}(x)$ fields whose coupling are given by:

$$
\mathscr{L}^{\operatorname{lnt}}(\beta)=\mathscr{L}^{\operatorname{lnt}}(x)+\beta^{\alpha}(x) \Delta_{N}^{\alpha}(x)
$$

The $\Delta_{N}(x)$ vertices affect the subtraction indices of a diagram in a way accounted for by assigning to the external $\beta^{\alpha}$ legs null U.V. dimension and I.R. dimension one. The W.I. are altered by the introduction of the $\beta^{\alpha}(x)$ fields as:

$$
\begin{aligned}
\mathscr{W}^{\alpha}(x) \Gamma(\beta)= & \frac{\delta}{\delta \beta^{\alpha}(x)} \Gamma(\beta) \\
& +N_{3}^{4}\left[\Delta^{\prime \alpha}(x, \beta)\right] \Gamma(\beta)+O(s-1)
\end{aligned}
$$

where the first term on r.h.s. stands for the insertion in $\Gamma(\beta)$ of an external $\Delta_{N}^{\alpha}(x)$ vertex and the second one indicates collectively all the remaining contribution implied from the Q.A.P.

We proceed to analyze both members of Eq. (69) in order to put into evidence the terms of least order in $\hbar^{1 / 2}$. Recalling our induction hypothesis $\left(N_{3}^{4}\left[\Delta^{\alpha}(x)\right] \Gamma=O\left(\hbar^{\overline{2}}\right), N \geqq 2\right)$ and on the basis of Theorem 1 (in particular $\lambda_{V}=1$ for the vertex $\left.\Delta_{N}^{\alpha}(x)\right)$ observe that the $\beta^{\alpha}(x)$ dependent terms in $\Gamma(\beta)$ are at least of the order $\hbar^{\frac{N}{2}}$ and that, moreover the least order contribution is given by the sole tree approximation term $\int d x \beta^{\alpha}(x) \Delta_{N}^{\alpha}(x)$. Hence we have:

$$
\frac{\delta}{\delta \beta^{\alpha}(x)} \Gamma(\beta)=\Delta_{N}^{\alpha}(x)+O\left(\hbar^{\frac{N+1}{2}}\right)
$$

and, taking into account Eq. (63) we get:

$$
\left.\mathscr{W}^{\alpha}(x) \Gamma(\beta)\right|_{s=1}=\Delta_{N}^{\alpha}(x)+\int d y \beta^{\gamma}(y) \mathscr{W}^{\alpha}(x) \Delta_{N}^{\gamma}(y)+O\left(\hbar^{\frac{N+1}{2}}\right) .
$$

Substituting these equations into Eq. (69) yields

$$
\begin{aligned}
N_{3}^{4}\left[\Delta^{\prime \alpha}(x, \beta)\right] \Gamma(\beta) & =\int d y \beta^{\gamma}(y) \mathscr{W}^{\alpha}(x) \Delta_{N}^{\gamma}(y)+O\left(\hbar^{\frac{N}{2}}\right) \\
& \equiv \Delta_{N}^{\prime \alpha}(x, \beta)+O\left(\hbar^{\frac{N+1}{2}}\right) .
\end{aligned}
$$

After all this preliminary work we can now finally obtain the consistency conditions for the breaking $\Delta_{N}^{\alpha}(x)$, arising from the commutation rules of the local Ward operators $\mathscr{W}^{\alpha}(x)$. From Eqs. (27), (69) we get

$$
\begin{aligned}
\left.\delta(x-y) f^{\alpha \beta \gamma} \mathscr{W}^{\gamma}(x) \Gamma(\beta)\right|_{S=1} & =\left.\frac{\delta}{\delta \beta^{\alpha}(x)} \Delta^{\prime \beta}(\beta, y) \Gamma(\beta)\right|_{s=1} \\
& =\left.\frac{\delta}{\delta \beta^{\beta}(y)} \Delta^{\prime \alpha}(x, \beta) \Gamma(\beta)\right|_{s=1} .
\end{aligned}
$$


Isolating the least order terms $\left(\hbar^{\frac{N}{2}}\right)$ in both members of Eq. (73) and substituting into its r.h.s. Eq. (72) we have [22],

$$
\delta(x-y) f^{\alpha \beta \gamma} \Delta_{N}^{\gamma}(x)=\mathscr{W}^{\alpha}(x) \Delta_{N}^{\beta}(y)-\mathscr{W}^{\beta}(y) \Delta_{N}^{\alpha}(x) .
$$

This equation has been discussed in purely algebraic terms in [10] under the hypothesis that the U.V. dimension of $\Delta_{N}^{\alpha}(x)$ does not exceed 4 . It turns out that its general solution is

$$
\Delta_{N}^{\alpha}(x)=\mathscr{W}^{\alpha}(x) \hat{\Delta}_{N}+\delta^{\alpha 0} \Delta_{N}^{0 \natural}(x)+\partial_{\mu} g^{\mu \alpha}(x)
$$

where $\hat{\Delta}_{N}=\int \hat{\Delta}_{N}(x) d x$, and both $\hat{\Delta}_{N}(x)$ and the other two contributions on the r.h.s. have U.V. dimensions $\leqq 4$. Moreover $\hat{\Delta}_{N}$ is defined up to gauge invariants, the term $\delta^{\alpha 0} \Delta_{N}^{0 \natural}(x)$ indicates a possible gauge invariant breaking to the W.I. relative to the abelian invariant subalgebra, and $\partial_{\mu} g^{\mu \alpha}(x)$ stands for the possible contribution of the Adler Bardeen anomaly [11].

In our model, where the Adler Bardeen anomaly is obviously absent, the presence of $\Delta_{N}^{0 \text { t }}(x)$ can also be excluded on the basis of the invariance of the lagrangian for the inversions in Eqs. (20). Indeed $\Delta_{N}^{0 \text { t }}(x)$ should be odd under these transformations, which is uncompatible with the gauge invariance and the U.V. dimensionality requirements. In other models, gauge invariant terms of this kind can in general be excluded by power counting arguments [10].

The last point we have to check in order to complete the proof of the Lemma is that the I.R. dimensionality of $\hat{\Delta}_{N}(x)$ in Eq. (62) is greater than or equal to four. In the notation of Sect. 2, possible contribution to $\hat{\Delta}$ violating this condition are:

$$
a \varphi_{0}(x)+b \varphi_{1}^{+}(x) \varphi_{1}^{-}(x)+c \psi_{0}^{2}(x) .
$$

Recalling that $\hat{\Delta}_{N}$ in Eq. (75) is determined up to gauge invariants, we are free to choose the coefficient $a$ in Eq. (76) equal to zero. To show that the coefficient $b$ vanishes we use Eqs. (61), (63) and obtain

$$
\begin{aligned}
& \quad-\left.i p_{\mu} \frac{\delta}{\delta \tilde{\varphi}_{1}^{+}(p)} \frac{\delta}{\delta \mathscr{A}_{\mu}^{-}(0)} \Gamma\right|_{\Phi=-0}+\left.\sqrt{3} F \frac{\delta}{\delta \tilde{\varphi}_{1}^{+}(p)} \frac{\delta}{\delta \varphi_{1}^{-}(0)} \Gamma\right|_{\Phi=0} \\
& \equiv G(p)=\sqrt{3} F b+O\left(\hbar^{\frac{N+1}{2}}\right)
\end{aligned}
$$

from which it follows that the least order term $\left(\hbar^{\frac{N}{2}}\right)$ of $G(p)$ is equal to $\sqrt{3} F b$. Since the renormalization rules enforce $G(0)=0$, it follows $b=0$. The same method applies straightforwardly to show that $c=0$ too.

\section{Conclusions}

We have so exhausted a systematic discussion of a perturbative approach to an Euclidean field theory with radiative "mass generation"; let us remark, as a final comment, that we have no idea about the existence of a corresponding perturbative structure for the $S$-matrix of the model, the reason being that the $S$ matrix elements contain mass-shell singularities which, according to the mechanism discussed in Sect. 3, could mix in a completely uncontrolled way the order of 
our perturbation expansions. However it is of some comfort to us the fact that is not the only sickness affecting the perturbative $S$-matrix in these kind of models since, in the presence of unstable particles, there is no perturbative framework, to our knowledge, giving a unitary $S$-matrix and preserving the W.I. [23].

Acknowledgements. One of us (C.B.) is deeply indebted to Prof's K. Symanzik, R. Stora, K. Pohlmeyer and to A. Rouet for helpful discussions during the draft of this paper.

\section{Appendix}

In this Appendix we analyze in detail the $\hbar^{1 / 2}$ degree of the amplitudes of our theory subtracted according to the rules given in Eqs. (35), (36); the analysis is further extended to include also possible insertions of composite external vertices in our Green functions in order to arrive at the results of Theorem 1, Sect. 3.

We shall proceed in two steps: the first one is to connect the behaviour for vanishing $\hbar$ of the amplitudes with that of the corresponding Feynman integrand. This evaluation is carried out with a method which is a straightforward generalization of the one employed by Zimmermann and Lowenstein [7] to discuss the convergence and power counting properties of Feynman integrals with massless propagators. The second step is to identify the leading order in $\hbar^{1 / 2}$ of the integrands; for this we shall adopt the same inductive procedure illustrated in [6].

Let us recall that the contribution to a Green function of a proper subtracted Feynman diagram has the general form

$$
\begin{gathered}
J(p)=\int d k R(p, k) \\
R(p, k)=\frac{P(p, k)}{\prod_{j=1}^{n}\left(l_{j}^{2}+m_{j}^{2}\right)^{n_{j}}}
\end{gathered}
$$

where all momenta are Euclidean, $k$ and $p$ denote collectively the integration variables $k_{1}, \ldots, k_{m}$ and the external momenta $\left(p_{1}, \ldots, p_{n}\right)$ fixed at a non exceptional value. The vectors $l_{j}$ are linear combinations of $k$ 's and $p$ 's, $P(p, k)$ is a polynomial in $p$ and $k$ and all the masses $m_{j}^{2}$ are greater than or equal to zero with the P.G.B. ones proportional to $\hbar$.

Let $u_{1}(k) \ldots u_{a}(k), v_{1}(k) \ldots v_{b}(k), a+b=m$ be a non singular change of basis in the linear space spanned by the $k$ 's; the hyperplane $H$ is define by the condition that $v_{1}(k) \ldots v_{b}(k)$ be kept constant. With this notation and with the definitions in Eq. (39), we will show that

$$
\underline{\operatorname{deg}}_{\hbar^{1 / 2}} J(p) \geqq \min _{\{\text {hyperplanes } H\}}\left[\underline{\operatorname{deg}}_{u \hbar^{1 / 2}} R(p, k)+4 a\right] .
$$

Concerning the integrand in Eq. (A.1b), we denote, after Ref. [7], by $S_{0}$ the set of lines with $m^{2}=0$ or $m^{2} \propto \hbar$; moreover let $S \subset S_{0}$ such that $l_{i} \in S, l_{j} \in S_{0}, l_{i}^{2}=l_{j}^{2}$ imply $l_{j} \in S$ and let $T$ stand for the complement of $S$.

Chosen an arbitrary number $r>0$, we define the subdomains

$$
\begin{aligned}
& D_{S} \equiv\left\{k: l_{i}^{2}(k) \leqq r \text { if } l_{i} \in S\right\} \\
& D_{T} \equiv\left\{k: l_{i}^{2}(k)>r \text { if } l_{i} \in T\right\}
\end{aligned}
$$


so that the integral in Eq. (A.1a) can be written as

$$
J=\sum_{\{S\}} J_{S}
$$

where

$$
J_{S}=\int_{D_{S} \cap D_{T}} d k R(p, k)
$$

The domain $D_{S}$ is further divided into disjoint sectors $D_{S \pi}$ where $\pi$ is any permutation of the lines in $S$ and

$$
D_{S \pi} \equiv\left\{k: l_{\pi_{l_{1}}}^{2} \leqq l_{\pi_{i_{2}}}^{2} \leqq \ldots \leqq l_{\pi_{i_{n}}}^{2} \leqq r\right\} .
$$

For each permutation $\pi$ we select a basis $u_{1}, \ldots, u_{a}$ of momenta of the $S$ lines choosing $u_{j}, 1 \leqq j \leqq a$, as the first, and hence the smallest among the momenta of the ordered chain in Eq. (A.5), which is independent from $u_{1}, \ldots, u_{j-1}$ and from the external momenta $p$.

Denoting by $v_{1}, \ldots, v_{b}, a+b=m$, the completion of this basis in the full $k$ space, the $P(p, k)$ polynomial in Eq. (A.1b) decomposes as [7]

$$
P(p, k)=\sum_{\alpha_{S}} M_{\alpha_{S}}(u) C_{\alpha_{S}}(v, p)
$$

where $M_{\alpha_{s}}(u)$ are monomials in the $u$ momenta containing all the powers of $\hbar$. It follows that:

$$
\begin{aligned}
\left|J_{S}\right| & \leqq \int_{D_{S} \cap D_{T}} d k|R(p, k)| \\
& \leqq \sum_{\{\hbar\}} \int_{D_{S \pi} \cap D_{T}} d k|R(p, k)| \leqq \sum_{\pi, \alpha} J_{S \pi \alpha}
\end{aligned}
$$

with

$$
J_{S \pi \alpha}=\int_{d_{S \pi}} d \mu \frac{\left|M_{\alpha_{S}}(u)\right|}{\prod_{l_{J}^{2} \in D_{S \pi}}\left(l_{j}^{2}+m_{j}^{2}\right)^{n_{j}}} \int_{d_{T}} d v \frac{\left|C_{\alpha_{S}}(v, p)\right|}{\prod_{l_{i}^{2} \in D_{T}}\left(l_{i}^{2}+m_{i}^{2}\right)^{n_{i}}}
$$

where the domains of the integration variables $u$ and $v$ are given by

$$
\begin{aligned}
& d_{S \pi}=\left\{u_{1}^{2} \equiv l_{11}^{2} \leqq l_{12}^{2} \leqq \ldots \leqq l_{1 k_{1}}^{2} \leqq u_{2}^{2} \equiv l_{21}^{2} \leqq l_{22}^{2}\right. \\
& \left.\quad \leqq \ldots \leqq l_{2 k_{2}}^{2} \leqq u_{3}^{2} \equiv l_{31}^{2} \leqq \ldots \leqq u_{a}^{2} \equiv l_{a 1}^{2} \leqq \ldots \leqq l_{a k_{a}}^{2} \leqq r\right\} \\
& \quad d_{T}=\left\{v \mid l_{j}^{2}(u, v, p)>r \text { for } l_{j} \in T\right\}
\end{aligned}
$$

Recalling that our subtraction procedure insures that the integral in Eq. (A.1a) is absolutely convergent, we can majorize the subintegral on the domain $d_{T}$ in Eq. (A.9) by the same method of Ref. [7] to obtain

$$
J_{S \pi \alpha} \leqq \Lambda_{S \pi \alpha}(p) \int_{d_{S \pi}} d u \frac{\left|M_{\alpha_{S}}(u)\right|}{\prod_{j}\left(l_{j}^{2}+m_{j}^{2}\right)^{n_{j}}} .
$$

Remark that if any $l_{j}^{2}$ in Eq. (A.10) depends upon the external momenta, then for a choice of $r>0$ sufficiently small, $J_{S \pi \alpha}$ vanishes since the domain of integration $d_{S \pi}$ is empty [7]; for this reason we shall, from now on, assume that the $l_{j}$ 's are linear combinations of the $u$ momenta alone. 
We now introduce explicitely $\hbar$ into our treatment by further subdividing $d_{S \pi}$ into disjoint domains $d_{S \pi l}$ where

$$
d_{S \pi l}=\left\{\left\{u_{1} \ldots u_{a}\right\} \in d_{S \pi}, u_{l-1}^{2} \leqq \hbar \leqq u_{l}^{2}\right\}
$$

Obviously only $d_{S \pi \alpha}$ will be non empty if $\hbar>r$; the integral in Eq. (A.10) can thus be decomposed according to

$$
J_{S \pi \alpha}=\Lambda_{S \pi \alpha}(p) \sum_{l=1}^{a} J_{S \pi \alpha l}
$$

where

$$
J_{S \pi \alpha l}=\int_{d_{S \pi l}} d u \frac{\left|M_{\alpha S}\right|}{\prod_{j}\left(l_{j}^{2}+m_{j}^{2}\right)^{n_{j}}} .
$$

Adopting the notation in Eq. (A.9a), the above integral is first majorized by substituting in the integrand the following inequalities:

$$
\frac{1}{l_{i k}^{2}+m_{i k}^{2}} \leqq \frac{2}{l_{i k}^{2}+u_{i-1}^{2}}
$$

when either $1 \leqq i \leqq l-1$ and $m_{i k} \equiv 0$ or $a \geqq i>l\left(u_{0}^{2}=0\right.$ by convention) and

$$
\frac{1}{l_{i k}^{2}+m_{i k}^{2}} \leqq \frac{\text { const }}{\hbar}
$$

when $1 \leqq i \leqq l-1$ and $m_{i k}^{2}=\gamma_{i k}^{2} \hbar>0$. The choice of the basis $u_{1}, \ldots, u_{a}$ insures, for $i \geqq l$, the decomposition

$$
l_{i_{k}}=\mathscr{V}_{i_{k}}\left(u_{l}, \ldots, u_{i}\right)+\mathscr{U}_{i_{k}}\left(u_{1}, \ldots u_{l-1}\right)
$$

with $\mathscr{V}_{i_{k}}$ non vanishing, from which it follows the inequality [7]

$$
\frac{\mathscr{V}_{i_{k}}^{2}+u_{i-1}^{2}}{l_{i_{k}}^{2}+u_{i-1}^{2}} \leqq 1+\frac{\left|U_{i_{k}}\right|}{\left|u_{i-1}\right|}+\frac{\mathscr{U}_{i_{k}}^{2}}{u_{i-1}^{2}}
$$

where the r.h.s. is uniformly bounded on the integration domain $d_{S \pi l}$. The above result implies that the r.h.s. of Eq. (A.14a) can be further majorized for $a \geqq i \geqq l$ by replacing

$$
\frac{1}{l_{i_{k}}^{2}+u_{i-1}^{2}} \leqq \frac{\text { const }}{\mathscr{V}_{i_{k}}^{2}+u_{i-1}^{2}} .
$$

The substitution of Eqs. (A.17), (A.14) into Eq. (A.13), after extending the domain of integration from $d_{S \pi}$ to the one defined by

$$
\left\{u_{1}^{2} \leqq u_{2}^{2} \leqq \ldots \leqq u_{l-1}^{2} \leqq \hbar \leqq u^{2} \leqq \ldots \leqq u_{a}^{2} \leqq r\right\},
$$

yields :

$$
\begin{aligned}
& J_{S \pi \alpha l} \leqq \text { const. } \int_{u_{1}^{2} \leqq \hbar} d u_{1} \frac{\left|M_{\alpha_{S} 1}\right|}{\prod_{i}\left(l_{1 i}^{2}\right)^{\mu i_{1}} \hbar^{v_{1}}} \cdots \int_{u^{2}-2 \leqq u^{2}-1 \leqq \hbar} d u_{l-1} \frac{\left|M_{\alpha_{S} l-1}\right|^{2}}{\prod_{i}\left(l_{l-1 i}^{2}+u_{l-2}^{2}\right)^{\mu_{i l-2}} \hbar^{v_{i-1}}} \\
& \int_{\hbar u^{2} \leqq r} d u_{l} \frac{\left|M_{\alpha_{S} l}\right|}{\prod_{i}\left(\mathscr{V}_{l i}^{2}+\hbar\right)^{n_{l i}}} \cdots \int_{u_{a-1}^{2} \leqq u_{a}^{2} \leqq r} d u^{a} \frac{\left|M_{\alpha_{S} l}\right|}{\prod_{i}\left(\mathscr{V}_{a i}^{2}+u_{a-1}^{2}\right)^{n_{a i}}}
\end{aligned}
$$


where, for $i \leqq l-1$, the indices $v_{i}$ refer to the contribution of the propagators with $m_{i}^{2}$ proportional to $\hbar$ and $\mu_{i}$ to that of the remaining ones. Now the integral in $d u_{1} \ldots d u_{l-1}$ obeys :

$$
\begin{gathered}
J_{s \pi \alpha l} \leqq \operatorname{Const}\left(\hbar^{1 / 2}\right)^{k=1} \sum_{\hbar \leqq u_{l} \leqq r} d u_{l} \frac{\left|M_{\alpha s l}\right|}{\prod_{i}\left(\mathscr{V}_{l i}+\hbar\right)^{n_{l i}}} \ldots \\
\quad \ldots \int_{u_{a}^{2}-1 \leqq u_{a}^{2} \leqq r} d u_{a} \frac{\left|M_{\alpha_{S a}}\right|}{\prod_{i}\left(\mathscr{V}_{a i}^{2}+u_{a-1}^{2}\right)^{n_{a i}}}
\end{gathered}
$$

where

$$
d_{k}=4+\underline{\operatorname{deg}}_{u \hbar^{1 / 2}} M_{\alpha_{s k}}-2 \sum_{i} n_{k i} .
$$

Setting

$$
\delta=\max _{1 \leqq j \leqq a}\left(0, \sum_{i=j}^{a} d_{i}\right)
$$

we arrive at

$$
\begin{gathered}
J_{S \pi \alpha l} \leqq \text { Const. }\left(\hbar^{1 / 2}\right)^{\sum_{k=1}^{l-l^{1} d_{k}}} \int_{\hbar \leqq u^{2}} d u_{l} \frac{\left|M_{\alpha_{s} l}\right|}{\prod_{i}\left(\mathscr{V}_{l i}^{2}+\hbar\right)^{n_{l l}}} \\
\ldots \int_{u_{a-1}^{2} \leqq u_{a}^{2} \leqq r} d u_{a} \frac{\left|M_{\alpha_{S} a}\right|}{\prod_{i}\left(\mathscr{V}_{a i}^{2}+u_{a-1}^{2}\right)^{n_{a i}}}\left|\frac{\sqrt{r}}{u_{a}}\right|^{\delta} .
\end{gathered}
$$

The last integral on the r.h.s. of Eq. (A.22) is evaluated by rescaling the integration variable $u_{a}=w_{a}\left|u_{a-1}\right|$ and by extending the integration domain, so that

$$
\begin{gathered}
J_{S \pi \alpha l} \leqq \text { Const. }\left(\hbar^{1 / 2}\right)^{\sum^{l} \sum^{l}=d_{1}^{1} d_{k}} \int_{\hbar \leqq u^{2}} d u_{l} \frac{\left|M_{\alpha_{s} l}\right|}{\prod_{i}\left(\mathscr{V}_{l i}^{2}+\hbar\right)^{n_{l l}}} \\
\ldots\left|u_{a-1}\right|^{d_{a}-\delta} \int_{1 \leqq w_{a} \leqq \infty} \frac{d w_{a}}{\left|w_{a}\right|^{\delta}} \frac{\left|M_{\alpha_{S} a}\right|}{\prod_{i}\left(\mathscr{V}_{a i}^{2}+1\right)^{n_{a i}}} .
\end{gathered}
$$

The same technique, applied to the other terms, finally gives

$$
J_{S \pi \alpha l} \leqq \operatorname{Const}\left(\hbar^{1 / 2}\right)^{\left(\sum_{k=1}^{a} d_{k}\right)-\delta} \text {. }
$$

The result anticipated in Eq. (A.2) is obtained by comparing the exponent of $\hbar^{1 / 2}$ in Eq. (A.24) with the behaviour of the integrand $R(p, k)$; indeed we have that

$$
\sum_{k=1}^{a} d_{k}-\delta \equiv d=\sum_{i=1}^{l-1} d_{i}-\min _{l \leqq j \leqq a} \sum_{k=l}^{i} d_{k}
$$

and, from Eqs. (A.7), (A.18)

$$
\begin{aligned}
& \underline{\operatorname{deg}}_{u \hbar^{1 / 2}} P(p, k)=\min _{\left\{\alpha_{S}\right\}} \operatorname{deg}_{u \hbar^{1 / 2}} M_{\alpha_{S}} \\
& \leqq \underline{\operatorname{deg}}_{u \hbar^{1 / 2}} M_{\alpha_{S}}=\sum_{k=1}^{a} \underline{\operatorname{deg}_{u \hbar^{1 / 2}} M_{\alpha_{S} k}}
\end{aligned}
$$


which substituted into Eq. (A.15) yields,

$$
d \geqq 4 a+\underline{\operatorname{deg}}_{u \hbar^{1 / 2}} R(p, k) .
$$

Once the first connection is made, we proceed to evaluate the r.h.s. of Eq. (A.27). To this purpose we recall briefly the notion of complete forest.

Given a connected diagram $\Lambda$ and a hyperplane $H$ in the linear space spanned by the $k$ 's, let $\bar{\gamma}$ be a reduced subdiagram of $\Lambda$ (i.e. a diagram $\gamma \subset \Lambda$ with a set of 1.P.I., mutually disjoint $\lambda_{i} \subset \gamma$ contracted to a point); we shall say that $\bar{\gamma}$ lies along $H$ (in symbols $\bar{\gamma} \| H$ ) if all the internal lines of $\bar{\gamma}$ have integration momenta belonging to $H$ : if no line of $\bar{\gamma}$ satisfies this condition $\bar{\gamma}$ will be called skew to $H(\bar{\gamma} \times H)$.

A $\Lambda$-forest $C$ is said to be complete with respect to $H$ if:

i) $\Lambda$ 1.P.I $\Rightarrow \Lambda \in C$

ii) $\forall \gamma \in C \cup\{\Lambda\}$ it follows either $\bar{\gamma}(C) \| H$ or $\bar{\gamma}(C) \nmid H$ where $\bar{\gamma}(C)$ is obtained by reducing $\gamma$ with respect to all the elements of $C$ properly contained in it.

In terms of the set of complete forests $\mathscr{C}_{H}(\Lambda)$ the subtracted integrand in Eqs. (35) can be rewritten as

$$
\begin{aligned}
R_{\Lambda} & =\sum_{C \in \mathscr{C}_{H}(\Lambda)} R_{\Lambda}(C) \\
R_{\Lambda}(C) & =\left(1-\tau_{\Lambda}\right) Y_{\Lambda}(C)
\end{aligned}
$$

where $Y_{\gamma}(C), \gamma \subset \Lambda$ is defined recursively by

$$
\begin{aligned}
& Y_{\gamma}(C)=I_{\bar{\gamma}(C)} S_{\gamma} \prod_{\alpha} f_{\gamma_{\alpha}} Y_{\gamma_{\alpha}}(C) \\
& f_{\gamma_{\alpha}}=\left\{\begin{array}{lll}
1-\tau_{\gamma_{\alpha}} & \text { if } \bar{\gamma}_{\alpha}(C) \chi H, & \bar{\gamma}_{\alpha}(C) \| H \\
-\tau_{\gamma_{\alpha}} & \text { otherwise }
\end{array}\right.
\end{aligned}
$$

and the subtraction operator $\tau_{\gamma}$ obeys the rules given in Eqs. (36), (37).

The whole analysis is centered on the structure of the integrand $Y_{\gamma}(C)$ which, choosing a basis $\left\{u_{1}, \ldots, u_{a}\right\}$ in $H$ and its completion $\left\{v_{1}, \ldots, v_{b}\right\}$, is of the type:

$$
Y_{\gamma}(C)=\frac{\sum_{j=1}^{n} f_{j}(p, s-1, \hbar) g_{j}(u, v, \ln \hbar)}{D(p, s-1, u, v, \hbar)}
$$

where for all $j, f_{j}(p, s-1, \hbar)$ is a monomial of degree $\mu_{j}$ in $\hbar^{1 / 2}$ and $v_{j}$ in $p, s-1$ and $g_{j}(u, v, \ln \hbar)$ is a polynomial which, according to Eq. (41) does not depend on $\ln \hbar$ when $\mu_{k}=2$. Moreover $D$ is a product of factors $\left[\mathscr{P}_{L_{i}}(p)+\mathscr{U}_{L_{i}}(u)+\mathscr{V}_{L_{i}}(v)\right]^{2}+M_{L_{i}}^{2}(s)$ corresponding to the line $L$ of the graph; in particular $\mathscr{V}_{L_{i}}(v) \equiv 0$ if $L \in \bar{\gamma}(C) \| H$, $\mathscr{V}_{L_{i}}(v)>0$ if $L \in \bar{\gamma}(C) \nmid H$ and $M_{L_{i}}^{2}(s)$ may vanish independently of $s$ or be proportional to $\hbar$ if $L$ belongs to a subtracted diagram. On the other hand $M_{L_{i}}^{2}(s)$ remains positive and order zero in $\hbar$ for $0 \leqq s<1$ if $L \in \bar{\gamma}(C)$ or $L \in \bar{\lambda}(C), \lambda$ being a maximal element of $C$ contained in $\gamma$ with $\bar{\lambda}(C) \chi H, \bar{\gamma}(C) \| H$.

Now, in complete analogy with the results and by the same method of Ref. [6], these specifications and the study of the $\hbar^{1 / 2}$ degree of $Y_{\gamma}(C)$ in Eq. (A.30) link the behaviour of the subtracted and unsubtracted diagrams, as follows. 
Lemma 1. Let $\tau_{\gamma}$ be the subtraction operator defined in Eqs. (36), (37); for any $C \ni \gamma \subset \Lambda, \gamma \neq \gamma_{2}\left(\gamma_{2}\right.$ a P.G.B. mass correction), we have

$$
\begin{aligned}
& \underline{\operatorname{deg}}_{\hbar^{1 / 2} u p(s-1)} \tau_{\gamma} Y_{\gamma} \min \left(\underline{\operatorname{deg}}_{\hbar^{1 / 2} u p(s-1)} Y_{\gamma}, \underline{\operatorname{deg}}_{\hbar^{1 / 2} u} Y_{\gamma}+\max \left(0, \varrho_{\gamma}\right)\right) \\
& \underline{\operatorname{deg}}_{\hbar^{1 / 2} u} \tau_{\gamma} Y_{\gamma} \geqq\left\{\begin{array}{l}
\min \left(\underline{\operatorname{deg}}_{\hbar^{1 / 2} u} Y_{\gamma}, \underline{\operatorname{deg}}_{\hbar^{1 / 2} u p(s-1)} Y_{\gamma}+1-\varrho_{\gamma}\right) \\
\text { if } \bar{\gamma}(C) \| H \\
\underline{\operatorname{deg}}_{\hbar^{1 / 2} u} Y_{\gamma} \text { if } \bar{\gamma}(C) \chi H
\end{array}\right. \\
& \underline{\operatorname{deg}}_{\hbar^{1 / 2} u p(s-1)}\left(1-\tau_{\gamma}\right) Y_{\gamma} \geqq \underline{\operatorname{deg}}_{\hbar^{1 / 2} u} Y_{\gamma}+\max \left(0, \varrho_{\gamma}\right) \text { if } \bar{\gamma}(C) \chi H
\end{aligned}
$$

while for the P.G.B. mass correction diagrams $\gamma \equiv \gamma_{2}$ we have

$$
\begin{aligned}
& \underline{\operatorname{deg}}_{\hbar^{1 / 2} u p(s-1)} \tau_{\gamma_{2}} Y_{\gamma_{2}} \geqq\left\{\begin{array}{l}
\min \left(\operatorname{deg}_{\hbar^{1 / 2} u p(s-1)} Y_{\gamma_{2}}, \operatorname{deg}_{\hbar^{1 / 2} u} Y_{\gamma_{2}}\right) \\
\gamma_{2} \text { non minimal } \bar{\gamma}_{2}(C) \| H \\
\min \left[\underline{\operatorname{deg}} \hbar_{\hbar^{1 / 2} u p(s-1)} Y_{\gamma_{2}}, \max \left(\underline{\operatorname{deg}}_{\hbar^{1 / 2} u} Y_{\gamma_{2}}, 3\right)\right] \\
\gamma_{2} \text { minimal }
\end{array}\right. \\
& \underline{\operatorname{deg}}_{\hbar^{1 / 2} u} \tau_{\gamma_{2}} Y_{\gamma_{2}} \geqq\left\{\begin{array}{l}
\min \left(\underline{\operatorname{deg}}_{\hbar^{1 / 2} u p(s-1)} Y_{\gamma_{2}}, \underline{\operatorname{deg}}_{\hbar^{1 / 2} u} Y_{\gamma_{2}}\right) \\
\text { if } \bar{\gamma}_{2}(C) \| H \\
\underline{\operatorname{deg}}_{\hbar^{1 / 2} u} Y_{\gamma_{2}} \text { if } \quad \bar{\gamma}_{2}(C) \nmid H
\end{array}\right. \\
& \operatorname{deg}_{\hbar^{1 / 2} u p(s-1)}\left(1-\tau_{\gamma_{2}}\right) Y_{\gamma_{2}} \geqq\left\{\begin{array}{l}
\operatorname{deg}_{\hbar^{1 / 2} u} Y_{\gamma_{2}} \bar{\gamma}_{2}(c) \chi H \text { non minimal } \\
\min \left(\operatorname{deg}_{\hbar^{1 / 2} u} Y_{\gamma_{2}}+1, \max \left(\operatorname{deg}_{\hbar^{1 / 2} u} Y_{\gamma_{2}}, 3\right)\right) \\
\bar{\gamma}_{2}(C) \Varangle H \text { minimal }
\end{array}\right.
\end{aligned}
$$

Using Lemma 1 and the recursive structure of the renormalized integrand corresponding to a given complete forest, the behaviour of any diagram can be obtained from that of the reduced (unsubtracted) graphs or the minimal ones, which is exhibited in the following:

Lemma 2. Given any subgraph $\gamma$ of $\Lambda$ let us define:

i) $N_{\gamma}=$ number of independent loops of $\gamma$

ii) $M_{\gamma(C)}=4 \sum_{\lambda \in C} N_{\bar{\lambda}(C) \| H} N_{\bar{\lambda}(C)}$

iii) $v_{\gamma}=\sum_{k} n_{k}(\gamma)\left(\underline{d}_{k}-\underline{d}_{k}^{0}\right)$

where $n_{k}(\gamma)$ is the number of $k$-th type external legs and $\underline{d}_{k}, \underline{d}_{k}^{0}$ are given in Eq. (38) and Eq. (42) respectively

$$
\begin{aligned}
& \text { iv) } \sigma_{\gamma}= \begin{cases}v_{\gamma} & \text { if } \quad \varrho_{\gamma} \geqq 0 \\
\max \left(0, \varrho_{\gamma}+v_{\gamma}\right) & \text { if } \quad \varrho_{\gamma}<0\end{cases} \\
& \text { v) } \lambda(\gamma)=\sum_{V \in \mathfrak{v}_{e}(\gamma)}\left[\frac{\varrho_{V}}{2}\right] \equiv \sum_{V \in \mathfrak{v}_{2}(\gamma)} \lambda_{V}
\end{aligned}
$$


where $\mathfrak{v}_{e}(\gamma)$ is the set of composite external vertices of $\gamma$ and $[x]$ means the least integer nearest to $x$.

With all these definitions we have:

a) $\underline{\operatorname{deg}}_{\hbar^{1 / 2} u p(s-1)} I_{\bar{\gamma}(C)}+M_{\bar{\gamma}(C)} \geqq 2 N_{\bar{\gamma}(C)}+\varrho_{\gamma}+v_{\gamma}$

$$
-\lambda_{\bar{\gamma}(C)}-\sum_{C \ni \gamma_{i} C \gamma}\left(\max \left(0, \varrho_{\gamma_{i}}\right)+\sigma_{i}\right) \quad \text { if } \quad \bar{\gamma}(C) \| H
$$

b) $\underline{\operatorname{deg}}_{\hbar^{1 / 2} u} I_{\bar{\gamma}(C)} \geqq 2 N_{\bar{\gamma}(C)}$.

In both cases the $\hbar$ dependence of the external vertices of $\gamma$ has not been taken into account.

Proof. Applying the definitions in Eqs. (43), (44), (A.30), we obtain when $\bar{\gamma}(C) \| H$

$$
\begin{aligned}
& \operatorname{deg}_{\hbar^{1 / 2} u p(s-1)} I_{\bar{\gamma}(C)}+M_{\bar{\gamma}(C)}=2 N_{\bar{\gamma}(C)}+4 \\
& \quad+\sum_{V \in \mathfrak{v}_{i}(\gamma)}\left(D_{V}-4\right)+\sum_{V \in \mathfrak{v}_{e}(\gamma)}\left(D_{V}-4\right)+\sum_{L \in \bar{\gamma}}\left(D_{L}+4\right)-\sum_{C \ni \gamma_{l} C \gamma}(4)
\end{aligned}
$$

where $2 N_{\bar{\gamma}(C)}$ is the loop factor and the last term on the r.h.s. is the contribution of the diagrams with respect to which $\gamma$ is reduced. Recalling the definition of $\varrho_{\gamma}$ and noting that each line ends in two vertices we get:

$$
\begin{aligned}
& \underline{\operatorname{deg}}_{\hbar^{1 / 2} u p(s-1)} I_{\bar{\gamma}(C)}+M_{\bar{\gamma}(C)} \geqq 2 N_{\bar{\gamma}(C)}+\varrho_{\gamma}+v_{\gamma} \\
& \quad+\sum_{V \in \mathbf{v}_{i}(\bar{\gamma})}\left(D_{V}+\sum_{k} v_{k}(V) \underline{d}_{k}^{0}-\varrho_{V}\right)+\sum_{V \in \mathfrak{v}_{e}(\bar{\gamma})}\left(D_{V}+\sum_{k} v_{k}(V) \underline{d}_{k}^{0}-\varrho_{V}\right) \\
& \quad+\sum_{\gamma_{i} \in \gamma_{i} \subset \gamma}\left(\sum_{k} v_{k}\left(\gamma_{i}\right) \underline{d}_{k}^{0}-4+\sum_{V_{i} \in \gamma_{i}}\left(4-\varrho_{V}\right)\right)
\end{aligned}
$$

from which Eq. (A.34) is readily obtained after the following remarks:

a) the summation on the internal vertices, according to the condition in Eq. (46) gives a positive contribution;

b) for the external vertices, from Eq. (41b), we find

$$
D_{V}+\sum_{k} v_{k}(V) \underline{d}_{k}^{0} \geqq \frac{1}{2}\left[D_{V}+\sum_{k} v_{k}(V) \underline{d}_{k}\right] \geqq \frac{\varrho_{V}}{2}
$$

hence

$$
D_{V}+\sum_{k} v_{k}(V) \underline{d}_{k}^{0}-\varrho_{V} \geqq-\frac{\varrho_{V}}{2}
$$

and since the 1.h.s. is an integer, we have the stronger inequality

$$
D_{V}+\sum_{k} v_{k}(V) \underline{d}_{k}^{0}-\varrho_{V} \geqq \lambda_{V}
$$


c) the last term on the 1.h.s. of Eq. (A.39) can be rewritten as

$$
4-\sum_{k} v_{k} \underline{d}_{k}^{0}+\sum_{V_{i} \in \gamma_{i}}\left(\varrho_{V_{i}}-4\right)=\varrho_{\gamma}+v_{\gamma}
$$

and from the definition of $\sigma_{\gamma}$ (Eq. (A.35) we get

$$
\max \left(0, \varrho_{\gamma}\right)+\dot{\sigma}_{\gamma} \geqq \varrho_{\gamma}+v_{\gamma} .
$$

For what concerns the proof of the second part of the Lemma, it is sufficient to note that the choice of the $(s-1)^{2} M^{2}$ factor in the propagators implies $\operatorname{deg}_{u} I_{\bar{\gamma}} \geqq 0$ so that the $\hbar^{1 / 2}$ contribution may only arise from the loop factor $\hbar^{N_{\bar{\gamma}}}$, thus yielding Eq. (A.35).

Combining the results of Lemma 1 and Lemma 2 we arrive at:

Lemma 3. The Green functions defined by the Eqs. $(\mathrm{A} .28,29)$ satisfy:

a) without insertions of composite external vertices

$$
\begin{array}{ll}
\operatorname{deg}_{\hbar^{1 / 2} u p(s-1)} \tau_{\gamma_{2}} Y_{\gamma_{2}} \geqq N_{\gamma_{2}}+2-M_{\gamma_{2}} \bar{\gamma}_{2}(C) \| H & \text { (A.39a) } \\
\operatorname{deg}_{\hbar^{1 / 2 u p(s-1)}}\left(1-\tau_{\gamma_{2}}\right) Y_{\gamma_{2}} \geqq N_{\gamma_{2}}+2-M_{\gamma_{2}} \bar{\gamma}_{2}(C) \chi H \\
\left.\frac{\operatorname{deg}_{\hbar^{1 / 2}} \tau_{\gamma_{2}} Y_{\gamma_{2}}}{\underline{\operatorname{deg}}_{\hbar^{1 / 2} u}\left(1-\tau_{\gamma_{2}}\right) Y_{\gamma_{2}}}\right\} \geqq N_{\gamma_{2}}+3-M_{\gamma_{2}} \text { if } \quad N_{\gamma_{2}}>1 \text { or } \geqq * 3-M_{\gamma_{2}} \text { if } \quad N_{\gamma_{2}}=1
\end{array}
$$

where, in general, $\geqq * x-M_{\gamma}$ means $\geqq x-M_{\gamma}$ if $M_{\gamma}>0$ and $\geqq x-1$ if $M_{\gamma}=0$; while for $\gamma \neq \gamma_{2}$ we have

$$
\begin{aligned}
& \underline{\operatorname{deg}}_{\hbar^{1 / 2} u p(s-1)} \tau_{\gamma} Y_{\gamma} \geqq N_{\gamma}+1-M_{\gamma}+\max \left(0, \varrho_{\gamma}\right) \quad \text { if } \quad \bar{\gamma}(C) \| H \\
& \underline{\operatorname{deg}}_{\hbar^{1 / 2 u p(s-1)}}\left(1-\tau_{\gamma}\right) Y_{\gamma} \geqq N_{\gamma}+1-M_{\gamma}+\max \left(0, \varrho_{\gamma}\right) \text { if } \bar{\gamma}(C) \chi H \\
& \left.\quad \frac{\operatorname{deg}_{\hbar^{1 / 2}} \tau_{\gamma} Y_{\gamma}}{\underline{\operatorname{deg}}_{\hbar^{1 / 2} u}\left(1-\tau_{\gamma}\right) Y_{\gamma}}\right\} \geqq * N_{\gamma}+2-M_{\gamma}
\end{aligned}
$$

b) when composite external vertices are present we have

$$
\begin{aligned}
\operatorname{deg}_{\hbar^{1 / 2} u p(s-1)} \tau_{\gamma} Y_{\gamma} & \leqq N_{\gamma}+1-M_{\gamma}+\max \left(0, \varrho_{\gamma}\right)+\sigma_{\gamma}-\lambda_{\gamma} \text { if } \bar{\gamma}(C) \| H \\
\underline{\operatorname{deg}}_{\hbar^{1 / 2}{ }^{1 p(s-1)}}\left(1-\tau_{\gamma}\right) Y_{\gamma} & \geqq N_{\gamma}+1-M_{\gamma}+\max \left(0, \varrho_{\gamma}\right)+\sigma_{\gamma}-\lambda_{\gamma} \\
\left.\frac{\operatorname{deg}_{\hbar^{1 / 2}} \tau_{\gamma} Y_{\gamma}}{\operatorname{deg}_{\hbar^{1 / 2} \nu^{\prime}}\left(1-\tau_{\gamma}\right) Y_{\gamma}}\right\} & \geqq N_{\gamma}+2-M_{\gamma}+\sigma_{\gamma}-\lambda_{\gamma}
\end{aligned}
$$

Proof. Suppose that, for each given complete forest $C$, Eqs. (A.39), (A.40), (A.41) are true for the minimal diagrams, which will be proved later; assuming that the 
same conditions hold for the maximal subdiagrams properly contained in $\gamma$, by Lemma 2 and Eq. (A.29a) we obtain for the diagram $\gamma$ itself in case a):

$$
\begin{aligned}
& \operatorname{deg}_{\hbar^{1 / 2} u p(s-1)} Y_{\gamma_{2}} \geqq N_{\gamma_{2}}+2-M_{\gamma_{2}}+\varrho_{\gamma_{2}} \\
& \underline{\operatorname{deg}}_{\hbar^{1 / 2} u} Y_{\gamma_{2}}\left\{\begin{array}{l}
\geqq N_{\gamma_{2}}+3-M_{\gamma_{2}} \text { if } \quad N_{\gamma_{2}}>1 \\
\geqq * 3-M_{\gamma_{2}} \text { if } N_{\gamma_{2}}=1
\end{array}\right. \\
& \underline{\operatorname{deg}}_{\hbar^{1 / 2} u p(s-1)} Y_{\gamma} \geqq N_{\gamma}+1-M_{\gamma}+\varrho_{\gamma} \\
& \operatorname{deg}_{\hbar^{1 / 2} u} Y_{\gamma} \geqq N_{\gamma}+2-M_{\gamma} .
\end{aligned}
$$

To verify these relations it is important to remark that in Eq. (A.33d) $\sigma_{\gamma} \leqq 2$. the equality holding only for diagrams of the $\gamma_{2}$ type, as follows from Eq. (A.38d). In much the same way in case b) we get

$$
\begin{gathered}
\operatorname{deg}_{\hbar^{1 / 2} u p(s-1)} Y_{\gamma} \geqq N_{\gamma}+1-M_{\gamma}+\varrho_{\gamma}+v_{\gamma}-\lambda_{\gamma} \\
\operatorname{deg}_{\hbar^{1 / 2} u} Y_{\gamma} \geqq N_{\gamma}+3+v_{\gamma}-\lambda_{\gamma}-M_{\gamma}
\end{gathered}
$$

as can easily be verified making use of the new bound $\sigma_{\gamma} \leqq \lambda_{\gamma}$ deduced from Eq. (A.38d). Now Eqs. (A.39), (A.40), (A.41) follow from the above inequalities and Lemma 1.

It remains to prove that they are true for minimal diagrams, i.e., those with no subtracted subdiagrams. In this case directly from Lemma 1 and Lemma 2 we have, when no composite external vertices are present (case a);

$$
\begin{aligned}
& \underline{\operatorname{deg}}_{\hbar^{1 / 2} u p(s-1)} \tau_{\gamma_{2}} Y_{\gamma_{2}} \geqq \min \left(2 N_{\gamma_{2}}+1-M_{\gamma_{2}}, 2 N_{\gamma_{2}}+1\right) \\
& \geqq N_{\gamma_{2}}+3-M_{\gamma_{2}} \quad \bar{\gamma}_{2}(C) \| H \\
& \underline{\operatorname{deg}}_{\hbar^{1 / 2} u} \tau_{\gamma_{2}} Y_{\gamma_{2}} \geqq\left\{\begin{array}{l}
2 N_{\gamma_{2}}+1-M_{\gamma_{2}} \geqq N_{\gamma_{2}}+2-M_{\gamma_{2}}, \quad \text { if } \quad \bar{\gamma}_{2}(C) \| H \\
2 N_{\gamma_{2}} \geqq N_{\gamma_{2}}+1, \quad \text { if } \quad \bar{\gamma}_{2}(C) \chi H
\end{array}\right. \\
& \operatorname{deg}_{\hbar^{1 / 2} u}\left(1-\tau_{\gamma_{2}}\right) Y_{\gamma_{2}} \geqq 2 N_{\gamma_{2}} \geqq N_{\gamma_{2}}+1 \\
& \underline{\operatorname{deg}}_{\hbar^{1 / 2} u p(s-1)}\left(1-\tau_{\gamma_{2}}\right) Y_{\gamma_{2}} \geqq 2 N_{\gamma_{2}}+1 \geqq N_{\gamma_{2}}+2 \quad \text { if } \quad \bar{\gamma}_{2}(C) \| H \\
& \underline{\operatorname{deg}}_{\hbar^{1 / 2} u p(s-1)} \tau_{\gamma} Y_{\gamma} \geqq 2 N_{\gamma}-M_{\gamma}+\max \left(0, \varrho_{\gamma}\right) \\
& \geqq N_{\gamma}+1-M_{\gamma}+\max \left(0, \varrho_{\gamma}\right), \quad \text { if } \quad \bar{\gamma}(C) \| H \\
& \underline{\operatorname{deg}}_{\hbar^{1 / 2} u p(s-1)}\left(1-\tau_{\gamma}\right) Y_{\gamma} \geqq 2 N_{\gamma}-M_{\gamma}+\max \left(0, \varrho_{\gamma}\right) \\
& \geqq N_{\gamma}+1-M_{\gamma}+\max \left(0, \varrho_{\gamma}\right), \quad \text { if } \quad \bar{\gamma}(C) \chi H \\
& \left.\begin{array}{l}
\frac{\operatorname{deg}_{h^{1 / 2} u} \tau_{\gamma} Y_{\gamma}}{\operatorname{deg}_{h^{1 / 2} u}\left(1-\tau_{\gamma}\right) Y_{\gamma}}
\end{array}\right\} \geqq * 2 N_{\gamma}-M_{\gamma}
\end{aligned}
$$

and in the case b):

$$
\begin{aligned}
& \underline{\operatorname{deg}}_{\hbar^{1 / 2} u p(s-1)} \tau_{\gamma} Y_{\gamma} \geqq 2 N_{\gamma}+\varrho_{\gamma}-M_{\gamma}+v_{\gamma}-\lambda_{\gamma} \quad \text { if } \quad \bar{\gamma}(C) \| H \\
& \operatorname{deg}_{\hbar^{1 / 2} u p(s-1)}\left(1-\tau_{\gamma}\right) Y_{\gamma} \geqq 2 N_{\gamma}+\max \left(0, \varrho_{\gamma}\right)-M_{\gamma}, \quad \text { if } \quad \bar{\gamma}(C) \nmid H \\
& \underline{\operatorname{deg}}_{\hbar^{1 / 2} u} \tau_{\gamma} Y_{\gamma}\left\{\begin{array}{l}
\geqq * 2 N_{\gamma}+1-M_{\gamma}+v_{\gamma}-\lambda_{\gamma} \text { for } \varrho_{\gamma}>0, \bar{\gamma}(C) \| H \\
\geqq 2 N_{\gamma} \text { otherwise }
\end{array}\right.
\end{aligned}
$$


The proof of the Lemma is completed by noticing that the inequalities in Eqs. (A.45), (A.46), (A.47) are at least as good as the ones we wanted to prove.

We have now introduced all the main ingredients to arrive at the evaluation of the $\hbar^{1 / 2}$ degree of the subtracted Feynman integrals, as given by the following

Theorem. The Feynman integral $J_{\Lambda}(p)$ corresponding to a connected diagram $\Lambda$, subtracted according to the rules specified in Eqs. (A.28), (A.29), satisfies:

a) $\operatorname{deg}_{\hbar^{1 / 2}} J_{\Lambda}(p) \geqq N_{\Lambda}+1$ if no composite external vertices are present

b) $\underline{\operatorname{deg}}_{\hbar^{1 / 2}} J_{\Lambda}(p) \geqq N_{\Lambda}+2-\lambda_{\Lambda}$ otherwise

Proof. From Eq. (A.2) it follows that the inequalities in Eqs. (A.48) hold true if they are verified by $\operatorname{deg}_{\hbar^{1 / 2} u} R_{A}+4 a$. Making use of Lemma 3 , the theorem can be directly verified for tadpole or vacuum bubble diagrams; in the other cases we shall employ Eq. (A.28b) and analyze separately $\underline{\operatorname{deg}}_{\hbar^{1 / 2} u(s-1)} Y_{\Lambda}$ and $\operatorname{deg}_{\hbar^{1 / 2} u(s-1)} \tau_{\Lambda} Y_{\Lambda}$.

Concerning $\underline{\operatorname{deg}}_{\hbar^{1 / 2} u(s-1)} \tau_{\Lambda} Y_{\Lambda}$ we have from Lemma 3 :

$\underline{\operatorname{deg}}_{\hbar^{1 / 2} u(s-1)} \tau_{\Lambda} Y_{\Lambda} \geqq \underline{\operatorname{deg}}_{\hbar^{1 / 2} u} \tau_{\Lambda} Y_{\Lambda}$

$\geqq\left\{\begin{array}{l}N_{A}+1-M_{A} \text { with no composite external vertices } \\ N_{A}+2-M_{A}-\lambda_{A} \text { in the presence of composite external vertices }\end{array}\right.$

The evaluation of $\operatorname{deg}_{\hbar^{1 / 2} u(s-1)} Y_{A}$ is carried out by using in analogy to [6] the augmented diagram $\hat{\Lambda}$ which is obtained from $\Lambda$ by adding a new leg ( $q$-line) to each external vertex and a new vertex $V_{\infty}$ where all the $q$-lines meet. Each $q$-line is assigned a propagator $\frac{1}{\left(q^{2}(p)+m^{2}\right)^{\mu}}$ with $\mu$ large enough so that for any subdiagram $\gamma$ of $\hat{\Lambda}$ containing $V_{\infty}$, the choice $\varrho_{\gamma}, \delta_{\gamma}<0$ should be possible.

Furthermore we have

$$
\underline{\operatorname{deg}}_{\hbar^{1 / 2} u(s-1)} Y_{\Lambda} \geqq \underline{\operatorname{deg}}_{\hbar^{1 / 2} u(s-1)} Y_{\hat{\Lambda}}
$$

since no $q$-line lies along $H$ due to the non-exceptionality requirement for the external momenta of $\Lambda$.

The evaluation of the r.h.s. of Eq. (A.50) is done separately for the case $\overline{\hat{\Lambda}} \| H$ and $\overline{\hat{\Lambda}} \Varangle H$. In the first case, owing to the fact that $\overline{\hat{\Lambda}}$ does not contain any $q$-line, we have directly from Eq. (A.29a)

$$
\begin{aligned}
\underline{\operatorname{deg}}_{\hbar^{1 / 2} u(s-1)} Y_{\hat{\Lambda}} \equiv & \underline{\operatorname{deg}}_{\hbar^{1 / 2} u p(s-1)} Y_{\hat{\Lambda}}=\underline{\operatorname{deg}}_{\hbar^{1 / 2} u p(s-1)} I \overline{\hat{\Lambda}} \\
& +\sum_{\gamma_{i} \neq \gamma_{\infty}} \underline{\operatorname{deg}}_{\hbar^{1 / 2} u p(s-1)} f_{\gamma_{t}} Y_{\gamma_{t}}+\underline{\operatorname{deg}}_{\hbar^{1 / 2} u p(s-1)} f_{\gamma_{\infty}} Y_{\gamma_{\infty}} .
\end{aligned}
$$

Here $\gamma_{\infty} \subset \hat{\Lambda}$ is the maximal element of $C$ containing all the $q$-lines and $V_{\infty}$, hence $\varrho_{\gamma_{\infty}}<0, \delta_{\gamma_{\infty}}<0$ and $Y_{\hat{\Lambda}}$ does not vanish only if $\gamma_{\infty} \nmid H, f_{\gamma_{\infty}} \equiv 1$. Let us also remark 
that all the possible composite external vertices of $\Lambda$ belong to $\gamma_{\infty}$. Thus using Eq. (A.41b) we find

$$
\begin{aligned}
\underline{\operatorname{deg}}_{\hbar^{1 / 2} u p(s-1)}\left(1-\tau_{\gamma_{\infty}}\right) Y_{\gamma_{\infty}} & \equiv \underline{\operatorname{deg}}_{\hbar^{1 / 2} u p(s-1)} Y_{\gamma_{\infty}} \\
& \geqq N_{\gamma_{\infty}}+1-M_{\gamma_{\infty}}-\lambda_{\Lambda}
\end{aligned}
$$

where $N_{\gamma_{\infty}}$ does not receive contributions from the loops containing $q$-lines. Moreover from Eq. (A.36)

$$
\begin{aligned}
\underbrace{}_{\operatorname{deg}_{\hbar^{1 / 2} u p(s-1)} I} I_{\hat{\Lambda}}+M_{\overline{\hat{\Lambda}}} \geqq & 2 N_{\overline{\hat{\Lambda}}}+\sum_{V}\left(\not D_{V}+\sum_{k} v_{k} \underline{d}_{k}^{0}-4\right) \\
& +\sum_{\gamma_{i} \neq \gamma_{\infty}}\left(\sum_{k} v_{k}\left(\gamma_{i}\right) \underline{d}_{k}^{0}-4\right)+\sum_{k} v_{k}\left(\gamma_{\infty}\right) \underline{d}_{k}^{0} \\
\geqq & 2 N_{\overline{\hat{\Lambda}}}+2-\sum_{\gamma_{i} \neq \gamma_{\infty}}\left[\max \left(0, \varrho_{\gamma_{i}}\right)+\sigma_{\gamma_{i}}\right]
\end{aligned}
$$

the second inequality following from Eqs. (A.45), (A.33d) and (A.38d) and from the fact that $\gamma_{\infty}$ has at least two external legs. Recalling now that $\sigma_{\gamma_{i}} \leqq 1$ if $\gamma_{i}$ is not a P.G.B. propagator correction in which case $\sigma_{\gamma_{2}}=2$ and substituting Eqs. (A.53), (A.52), (A.39), (A.40) into Eq. (A.51) we arrive at

$$
\underline{\operatorname{deg}}_{\hbar^{1 / 2} u(s-1)} Y_{\hat{\Lambda}} \equiv \underline{\operatorname{deg}}_{\hbar^{1 / 2} u p(s-1)} Y_{\hat{\Lambda}} \geqq N_{\Lambda}+3-M_{\Lambda}-\lambda_{\Lambda} \text {. }
$$

Considering the case $\overline{\hat{\Lambda}} \nmid H$, and hence $V_{\infty} \in \overline{\hat{A}}$, we have from Eq. (A.29a)

$$
\begin{aligned}
\underline{\operatorname{deg}}_{\hbar^{1 / 2} u(s-1)} Y_{\hat{\Lambda}} & =\underline{\operatorname{deg}}_{\hbar^{1 / 2} u(s-1)} I_{\overline{\hat{\Lambda}}}+\sum_{\gamma_{i}} \underline{\operatorname{deg}}_{\hbar^{1 / 2} u(s-1)} \tau_{\gamma_{i}} Y_{\gamma_{i}} \\
& \geqq \underline{\operatorname{deg}}_{\hbar^{1 / 2} u} I_{\overline{\hat{\Lambda}}}+\sum_{\gamma_{i}} \underline{\operatorname{deg}}_{\hbar^{1 / 2} u} f_{\gamma_{i}} Y_{\gamma_{i}} .
\end{aligned}
$$

The substitution of Eqs. (A.35), (A.39c), (A.40c) into the r.h.s., recalling that the loops containing $q$-lines do not contribute any $\hbar$ power to the counting, yields:

$$
\begin{aligned}
\underline{\operatorname{deg}}_{\hbar^{1 / 2} u(s-1)} Y_{\hat{\Lambda}} \geqq 2 N_{\bar{\Lambda}}+\sum_{\gamma_{i}}\left(N_{\gamma_{i}}+2-M_{\gamma_{i}}-\lambda_{\gamma_{i}}\right) \\
\geqq N_{\Lambda}+3-M_{\Lambda}-\lambda_{\Lambda} .
\end{aligned}
$$

By comparison of Eqs. (A.54), (A.56), (A.49) we have for any complete forest $C$ $\underline{\operatorname{deg}}_{\hbar^{1 / 2} u(s-1)} R_{\Lambda}(C)$

$$
\geqq \begin{cases}N_{A}+1-M_{A} & \text { with no composite external vertices. } \\ N_{A}+2-M_{A}-\lambda_{\Lambda} & \text { with composite external vertices. }\end{cases}
$$

The results in Theorem 1 are now obtained directly from Eq. (A.28a) and the observation that $4 a \geqq M_{A}$ with the equality holding for $M_{A}>0$. 
As a final comment we point out that in some instances these evaluations can be improved, in particular it is easy to verify that an one loop diagram containing a P.G.B. mass counterterm of degree $\chi$ in $\hbar^{1 / 2}(\chi>2)$ satisfies

$$
\begin{aligned}
& \operatorname{deg}_{\hbar^{1 / 2} u} \tau_{\gamma} Y_{\gamma} \geqq \chi+1 \\
& \text { deg }_{\hbar^{1 / 2}{ }_{u}} Y_{\gamma} \geqq \chi+2 .
\end{aligned}
$$

The above Eqs. (A.58) clearly imply that the corresponding Feynman integral verifies:

$$
\underline{\operatorname{deg}}_{\hbar^{1 / 2}} J(p) \geqq \chi+1 \text {. }
$$

\section{References}

1. Weinberg, S.: Phys. Rev. Lett. 29, 1698 (1972); Phys. Rev. D13, 974 (1976); Phys. Rev. D7, 2887 (1973)

2. Georgi, H., Glashow, S.: Phys. Rev. D7, 2457 (1973)

3. Georgi, H., Pais, A.: Phys. Rev. D10, 1246 (1974)

4. Higgs, P.W.: Phys. Lett. 12, 132 (1964) Englert, F., Brout, R.: Phys. Rev. Lett. 13, 321 (1964) Kibble, T.: Phys. Rev. 155, 1544 (1967)

5. Goldstone, J.: Nuovo Cimento 19, 154 (1961)

6. Lowenstein, J.H.: Commun. Math. Phys. 47, 53 (1976)

7. Lowenstein, J.H., Zimmermann, W.: Commun. Math. Phys. 44, 73 (1975)

8. Coleman, S.: Fun with $S U(3)$. In: High energy physics and elementary particles. I.C.P.T. Proceedings (1965)

9. De Dominicis, C.: J. Math. Phys. 4, 255 (1963)

Jona, G.: Lasinio - Nuovo Cimento 34, 1790 (1964)

Nambu, Y.: Phys. Lett. 26B, 626 (1968)

10. Becchi, C., Rouet, A., Stora, R.: Renormalizable theories with symmetry breaking. Preprint, Marseille 75/P, 734 (1975)

11. Adler, S.L., Bardeen, W.: Phys. Rev. 182, 1515 (1968)

Adler, S.L.: Perturbation theory anomalies. In: Lectures on elementary particles and quantum field theory. Deser, S., Grisaru, M., Pendleton, H. (eds.). Cambridge, Mass. : M.I.T. 1970 Bardeen, W.: Phys. Rev. 184, 1848 (1969)

12. Clark, T.E., Piguet, O., Sibold, K.: Nucl. Phys. B119, 292 (1977)

Bardeen, W.A., Piguet, O., Sibold, K.: Phys. Lett. 72 B, 231 (1977)

13. Bogoliubov, N.N.: Exp. Theor. Phys. (U.S.S.R.) 34, 58 (1958); Nuovo Cimento X, 794 (1958)

14. Symanzik, K.: Commun. Math. Phys. 18, 227 (1970)

15. Pohlmeyer, K.: Large momentum behaviour of the Feynman amplitudes in theory. Desy report $74 / 44$ (1974)

Trute, H.: Graphical rules for the diagonalization of the Feynman denominator. Desy report 74/36 (1974)

Pohlmeyer, K. : Large momentum behaviour of Feynman amplitudes. In : Lecture notes in physics, No. 39. Araki, H. (ed.). Berlin, Heidelberg, New York : Springer 1975

16. Bourbaki, N.: Eléments de mathématique XI. Algébre Ch. $4, \S 5$

17. Clark, T., Lowenstein, J.H. : Nucl. Phys. B113, 109 (1976)

18. Symanzik, K.: Massless $\Phi^{4}$ theory in $4-\varepsilon$ dimensions. Desy report $73 / 58$ (1973); Lett. Nuovo Cimento 8, 771 (1973). On some massless superrenormalizable and non renormalizable theories. In : Lectures notes in physics. Vol. 39. Araki, H. (ed.). Berlin, Heidelberg, New York : Springer 1975

19. Lam, Y.M.P.: Phys. Rev. D6, 2145 (1972); Phys. Rev. D7, 2943 (1973)

Lowenstein, J.H.: Commun. Math. Phys. 24, 1 (1971) 
20. Becchi, C., Rouet, A., Stora, R.: Renormalizable models with broken symmetry. In: renormalization theory. Velo, G., Wightman, A.S. (eds.). Dordrecht, Boston: D. Reidel Publishing Company 1976

21. Zimmermann, W.: Local operator products and renormalization. In: Quantum field theory. Deser, S., Grisaru, M., Pendleton, H. (eds.). Cambridge, Mass. : M.I.T. 1970

22. Wess, J., Zumino, B.: Phys. Lett. B37, 95 (1971)

23. Blasi, A., Collina, R.: Lett. Nuovo Cimento 14, 143 (1975)

Communicated by R. Stora

Received February 1, 1979 